University of New Hampshire

University of New Hampshire Scholars' Repository

8-2003

\title{
High-Frequency Volume and Boundary Acoustic Backscatter Fluctuations in Shallow Water
}

Timothy C. Gallaudet

Scripps Institution of Oceanography

Christian de Moustier

University of California - San Diego

Follow this and additional works at: https://scholars.unh.edu/ccom

Part of the Oceanography and Atmospheric Sciences and Meteorology Commons

\section{Recommended Citation}

T. C. Gallaudet and C. P. de Moustier, 'High-frequency volume and boundary acoustic backscatter fluctuations in shallow water', The Journal of the Acoustical Society of America, vol. 114, no. 2, p. 707, 2003.

This Journal Article is brought to you for free and open access by the Center for Coastal and Ocean Mapping at University of New Hampshire Scholars' Repository. It has been accepted for inclusion in Center for Coastal and Ocean Mapping by an authorized administrator of University of New Hampshire Scholars' Repository. For more information, please contact Scholarly.Communication@unh.edu. 


\title{
High-frequency volume and boundary acoustic backscatter fluctuations in shallow water
}

\author{
Timothy C. Gallaudet and Christian P. de Moustier ${ }^{\mathrm{a}}$ \\ Marine Physical Laboratory, Scripps Institution of Oceanography, Mail Code 0205, \\ La Jolla, California 92037-0205
}

(Received 27 November 2001; revised 24 March 2003; accepted 21 April 2003)

\begin{abstract}
Volume and boundary acoustic backscatter envelope fluctuations are characterized from data collected by the Toroidal Volume Search Sonar (TVSS), a $68 \mathrm{kHz}$ cylindrical array capable of $360^{\circ}$ multibeam imaging in the vertical plane perpendicular to its axis. The data are processed to form acoustic backscatter images of the seafloor, sea surface, and horizontal and vertical planes in the volume, which are used to attribute nonhomogeneous spatial distributions of zooplankton, fish, bubbles and bubble clouds, and multiple boundary interactions to the observed backscatter amplitude statistics. Three component Rayleigh mixture probability distribution functions (PDFs) provided the best fit to the empirical distribution functions of seafloor acoustic backscatter. Sea surface and near-surface volume acoustic backscatter PDFs are better described by Rayleigh mixture or log-normal distributions, with the high density portion of the distributions arising from boundary reverberation, and the tails arising from nonhomogeneously distributed scatterers such as bubbles, fish, and zooplankton. PDF fits to the volume and near-surface acoustic backscatter data are poor compared to PDF fits to the boundary backscatter, suggesting that these data may be better described by mixture distributions with component densities from different parametric families. For active sonar target detection, the results demonstrate that threshold detectors which assume Rayleigh distributed envelope fluctuations will experience significantly higher false alarm rates in shallow water environments which are influenced by near-surface microbubbles, aggregations of zooplankton and fish, and boundary reverberation. (c) 2003 Acoustical Society of America.
\end{abstract}

[DOI: 10.1121/1.1588656]

PACS numbers: 43.30.Gv, 43.30.Vh, 43.30.Re [DLB]

\section{INTRODUCTION}

Validating statistical reverberation models is difficult because reverberation fluctuations are so strongly influenced by the sonar's beam pattern and spatial distribution of scatterers. ${ }^{1-6}$ The former is usually known through system calibration, but the latter is more difficult to characterize. Acoustic and optical imaging methods have been used for this purpose, mostly for studies of the seafloor, ${ }^{7-9}$ although some studies of the volume ${ }^{10}$ and sea surface ${ }^{11}$ have been performed. To the best of our knowledge, no such study has been conducted as a function of angle with a high resolution multibeam sonar measuring simultaneously seafloor, sea surface, and volume acoustic backscatter and reverberation. Such a study is warranted because rarely can reverberation be considered a single component process. Here, "single component scattering process" refers to a process dominated by acoustic backscatter from one type of scatterer, such as the sea floor, whereas "two-component scattering process" refers to a process dominated by acoustic backscatter from two types of scatterers, such as both boundaries, or a single boundary and biologic scatterers in the volume. Similarly, a three-component process refers to a process dominated by three types of scatterers, such as both boundaries and nearsurface bubbles, etc.

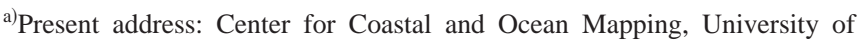
New Hampshire, 24 Colovos Road, Durham, NH 03824.
}

A recent study of seafloor reverberation process was performed by Lyons and Abraham, ${ }^{7}$ who found the threecomponent Rayleigh mixture distribution to be the most robust in describing observed fluctuations in seafloor acoustic backscatter amplitude data from a wide variety of seafloor types identified with optical and in situ sampling techniques. Here, we perform a similar study, but add to their results by (1) also including the log-normal probability distribution in the model-data comparisons, (2) analyzing data collected on a moving platform, thereby incorporating the influence of spatial variability on the backscatter amplitude fluctuations, (3) analyzing data from both boundaries and the volume, and (4) using coincident multibeam acoustic backscatter imagery to link the spatial distributions of various scatterers to the observed fluctuation statistics.

The data used in this study were collected by the Toroidal Volume Search Sonar (TVSS), a $68 \mathrm{kHz}$ cylindrical array which was deployed on a towfish at a depth of $78 \mathrm{~m}$ in waters $200 \mathrm{~m}$ deep, $735 \mathrm{~m}$ astern of a towship during engineering tests conducted by the U.S. Navy's Coastal System Station (CSS), Panama City, Florida (Fig. 1). The multibeam acoustic data collected by the TVSS were processed to construct boundary ${ }^{12,13}$ and volume ${ }^{14}$ acoustic backscattering strength images in horizontal and vertical planes around the towfish (Fig. 2). Here, we examine the statistics of, and fit probability distributions to the backscatter amplitudes corresponding to these data. The multibeam acoustic backscatter imagery provides the means for discriminating between vari- 


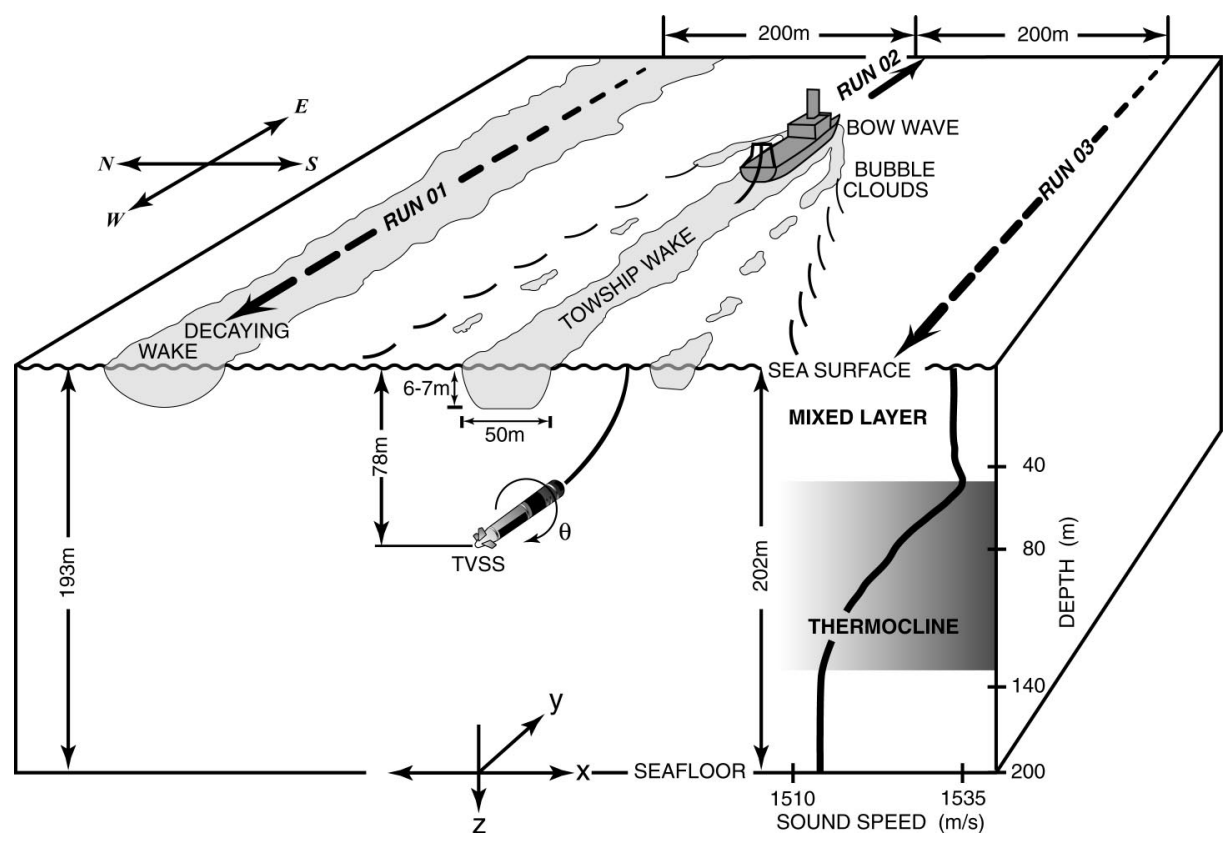

FIG. 1. Depiction of the TVSS deployment of 9 November 1994. Although each of the three parallel runs consisted of over 800 pings, the data presented in this paper are processed from only 100 pings in each of the three runs. The environmental conditions are summarized in the text, and more complete descriptions and analyses are presented in Refs. 12-14.

ous reverberation components and directly attributing nonhomogeneous spatial distributions of scatterers, such as bubbles, zooplankton, and multiple boundary interactions to non-Rayleigh backscatter amplitude distributions.

A useful model for understanding the statistical properties of acoustic reverberation is the point scattering model, ${ }^{15-20}$ which assumes that the total backscattered signal is the sum of $n$ replicas of the transmitted signal $s(t)$ backscattered from a homogeneous distribution of point reflectors

$$
F(t)=\sum_{i=1}^{n} a_{i} B\left(t_{i}\right) s\left(t-t_{i}, \xi_{i}\right)
$$

where $t_{i}$ is the time of arrival from the $i$ th scatterer, $a_{i}$ is the stochastic amplitude which corresponds to that scatterer's acoustic cross section, $B\left(t_{i}\right)$ describes the sonar's acoustic geometry and gain, and $\xi_{i}$ is a set of stochastic parameters defining the characteristics of the scattered signals, which may depend upon the relative motion between the acoustic array and the scatterers, their physical properties, and their spatial distribution.

In general, $F(t)$ will fluctuate around some time-varying mean value, and the quadrature components of the fluctuating part may be expressed as ${ }^{16}$

$$
V_{I, Q}(t)=F(t) / g(t),
$$

where $g(t)$ is the transient function whose reciprocal transforms the nonstationary reverberation sum in Eq. (1) to the stationary form $V_{I, Q}(t)$. This fluctuating signal, and its corresponding envelope are important because their probability density functions (PDFs) are used as the noise models against which target detection algorithms must operate. ${ }^{21}$

The model in (1) and (2) assumes that the number $n$ is governed by a Poisson distribution, where the scatterers producing the resulting reverberation are discrete, statistically independent in position, and homogeneously distributed within the sonar's resolution cell. If the number of scatterers in a single resolution cell is very large, and their scattering coefficient distribution $\left(a_{i}\right)$ is such that no small number of them contributes significantly to the reverberation energy, application of the central limit theorem results in a Gaussian distribution for $V_{I, Q}(t)$, with a Rayleigh distributed envelope and uniformly distributed phase.

In typical shallow water environments, the distributions of scatterers can rarely be assumed to be homogeneous, and different types of scatterers distributed on different spatial scales tend to produce more extreme reverberation values, depending upon the density of scatterers relative to the sonar's resolution cell size. For envelope fluctuation distributions, these may appear as multiple modes and/or large tails, deviating significantly from the traditional Rayleigh PDF. ${ }^{7,8,22-24}$

The distribution models considered in this study are the Rayleigh, $K$, Weibull, log-normal, and Rayleigh-mixture distributions. We chose these because (1) they are commonly used in underwater acoustics, (2) they have been observed in previous studies of volume and boundary backscatter and reverberation, and (3) some have been analytically related to the physical scattering mechanisms which produce them. Although a number of probability distribution models have been developed for specific boundary or volume reverberation conditions, $1,3,10,25,26$ our objective is to determine whether there is a common model flexible enough to describe both boundary and volume backscatter arising from nonhomogeneous, or patchy scatterer distributions that are typical in shallow water.

We begin in Sec. II with a description of the PDF models used in this study. Section III describes the TVSS signal processing methods and the data preparation steps. The results are described in Sec. IV, and we assess in Sec. V the physical mechanisms influencing these results and their implications for target detection.

\section{PROBABILITY DISTRIBUTION MODELS}

Each of the distribution functions discussed here may be represented as a function of one or several parameters that 

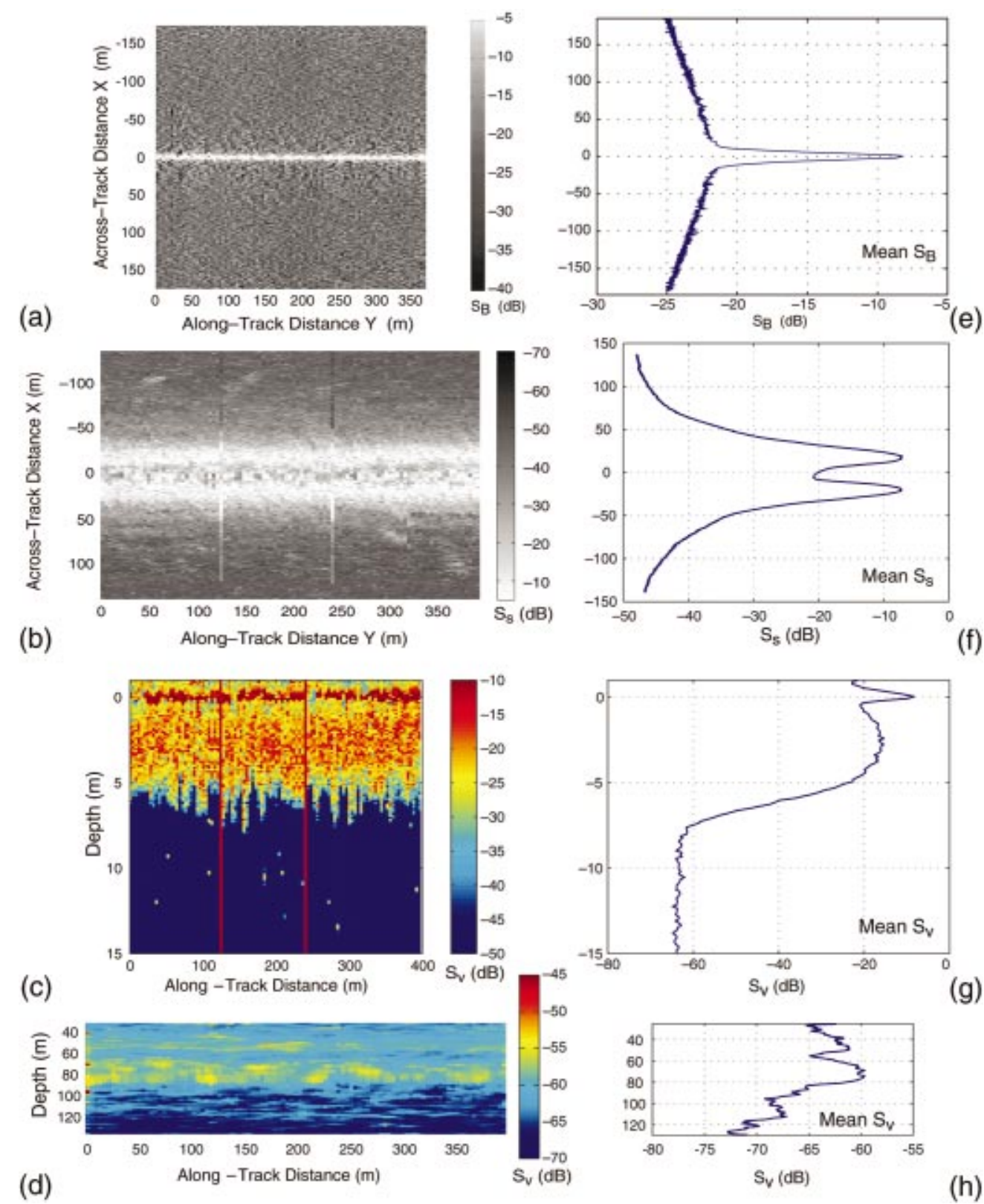

(g)

(h)

FIG. 2. TVSS-derived acoustic backscattering strength images displayed in coordinates relative to the towfish (a)-(d) and their corresponding along-track averages (e)-(h). (a) Bottom acoustic backscattering strength $\left(S_{B}\right)$ : The normal incidence return extends along-track near the center in the seafloor image and results from the natural angular dependence function of the silt and sand sediments in the region (Ref. 12). (b) Sea surface acoustic backscattering strength $\left(S_{S}\right)$ : The feature near the track center extending along-track in the sea surface backscattering strength image is influenced by vertical attenuation through bubbles in the towship's wake (Fig. 1) (Ref. 13). The moderately high backscattering strength features 50-100 m to the right and left of the track centerline are due to resonant scattering from bubble clouds generated by breaking ship waves. The two across-track lines near $Y=120 \mathrm{~m}$ and $235 \mathrm{~m}$ in this image and the vertical lines in (c) at the same along-track locations are corrupted data and were excluded from the analysis. (c), (d) Volume acoustic backscattering strength $\left(S_{V}\right)$ : The vertical volume backscattering strength image (c) formed by using the upward looking beams shows that the bubble layer associated with the towship's wake varies in scattering strength and depth along-track. The vertical volume image (d) formed in the vertical plane $47 \mathrm{~m}$ to the right of the TVSS shows the presence of volume scattering layers in the mixed layer and upper thermocline (Ref. 14).

must be estimated from the observed amplitude data, $A$ $=\left\{A_{1} A_{2} \cdots A_{N}\right\}$, whose samples are assumed to be independent and identically distributed. For parameter estimation, we use maximum likelihood estimation and the method of moments as described and implemented by Abraham. ${ }^{27}$

We start with the Rayleigh PDF for acoustic reverberation of amplitude $A \geqslant 0$ :

$$
p_{R}(A)=\frac{2 A}{\lambda_{R}} e^{-A^{2} / \lambda_{R}}
$$

and its cumulative distribution function $(\mathrm{CDF})$

$$
P_{R}(A)=1-e^{-A^{2} / \lambda_{R}}
$$

where $\lambda_{R}=\left\langle A^{2}\right\rangle$, with \langle\rangle representing the expected value. It describes reverberation whose in-phase and quadrature components are normally distributed with zero mean, and results from enough scatterers in the sonar's resolution cell for the central limit theorem to hold. ${ }^{16}$ The Rayleigh distribution has been observed for high frequency backscatter and reverberation from the seafloor, ${ }^{7}$ sea surface, ${ }^{28}$ and volume, ${ }^{10,29}$ and is 
a limiting case of the Ricean distribution in which scattering is primarily incoherent. ${ }^{30}$ Stanton ${ }^{30}$ has related the Rayleigh PDF of seafloor acoustic backscatter amplitude to the rms roughness and correlation area of the bottom.

The $K$ distribution may be represented as the product of a rapidly fluctuating, Rayleigh-distributed random variable, and a slowly varying, chi-distributed variable ${ }^{31-33}$ Its PDF is $^{27}$

$$
p_{K}(A)=\frac{4}{\sqrt{\alpha} \Gamma(\nu)}\left(\frac{A}{\sqrt{\alpha}}\right)^{\nu} K_{\nu-1}\left(\frac{2 A}{\sqrt{\alpha}}\right),
$$

and its $\mathrm{CDF}$ is

$$
P_{K}(A)=1-\frac{1}{\Gamma(\nu) 2^{\nu-1}}\left(2 \frac{A}{\sqrt{\alpha}}\right)^{\nu} K_{\nu}\left(\frac{2 A}{\sqrt{\alpha}}\right),
$$

with $A \geqslant 0 . K_{\nu-1}$ is the $\nu-1$ order modified Bessel function and $\Gamma($ ) is the gamma function. When the scale $1 / \sqrt{\nu}$ is applied to $A$, the Rayleigh distribution with power $\alpha$ is obtained, in the limit as $\nu$ tends to infinity, from the $K$ distribution. ${ }^{27}$ The $K$ distribution has been used to describe radar sea surface clutter because it has a direct physical interpretation: the Rayleigh component, with relatively short correlation widths, results from the many scattering contributions within the resolution cell that arise from small scale facets on the sea surface, whereas the chi-distributed component, with relatively long correlation widths, arises from the larger scale, mean sea surface tilt (e.g., swell). The $K$ distribution also has been used to describe signal envelope fluctuations in wireless channels ${ }^{34}$ and seafloor acoustic backscatter in sidescan sonar images. ${ }^{8,22}$

The Weibull distribution also is related to the Rayleigh distribution and has been used to describe seafloor backscatter amplitude distributions. ${ }^{7}$ The two-parameter Weibull PDF is $^{27}$

$$
p_{W}(A)=\alpha \beta A^{\beta-1} e^{-\alpha A^{\beta}}
$$

for $A \geqslant 0$, with its CDF given by

$$
P_{W}(A)=1-e^{-\alpha A^{\beta}},
$$

where it can be seen that the Rayleigh distribution results when $\beta=2$ and $\alpha=1 / \lambda_{R}$.

Whereas the $K$ and Weibull distributions may be related to physical scattering mechanisms through their relationships with the Rayleigh distribution, the log-normal distribution has yet to reveal such analytical connections. Nevertheless, the log-normal distribution has been observed in studies of underwater acoustic backscatter and propagation, ${ }^{23,35,36}$ radar clutter from the sea surface, ${ }^{37}$ and signal envelope fluctuations in wireless channels. ${ }^{34,38}$ The two-parameter lognormal PDF is ${ }^{39}$

$$
p_{L N}(A)=\frac{1}{\sqrt{2 \pi} \alpha A} e^{-(\ln A-\beta)^{2} / 2 \alpha^{2}}
$$

for $A>0$. It has the property that $\ln (A)$ is normally distributed with mean $\beta$ and variance $\alpha^{2}$. The log-normal CDF is

$$
P_{L N}(A)=\Phi\left(\frac{\ln A-\beta}{\alpha}\right),
$$

where

$$
\Phi(u)=\frac{1}{\sqrt{2 \pi}} \int_{-\infty}^{u} e^{-w^{2} / 2} d w
$$

is the CDF of a standard normal random variable $u$. Another property of the log-normal distribution is that if $A$ is $\log$ normally distributed, so is $A^{2}$; i.e., if the echo amplitude PDF has the form of Eq. (9), so will the PDF of the echo intensity. ${ }^{39}$

In typical shallow water environments, acoustic backscatter and reverberation result from several independent scattering mechanisms, such as bubbles, bioacoustic scatterers, and boundary roughness, and each of these may be characterized by different spatial scales. For high resolution, narrow beam sonars used in bioacoustic studies, multibeam bathymetric surveys, studies of near surface physical processes, and mine-countermeasures, the echo from a given resolution cell typically, though not necessarily, contains only one type of scatterer. Therefore, it is reasonable to consider that reverberation in such a scenario might be represented by a mixture of $m$ Rayleigh random variables, each with a component probability $\varepsilon_{i}$ and power $\lambda_{R, i}$. The resulting Rayleigh mixture PDF is ${ }^{27}$

$$
p_{R M}(A)=\sum_{i=1}^{m} \varepsilon_{i} \frac{2 A}{\lambda_{R, i}} e^{-A^{2} / \lambda_{R, i}},
$$

and its $\mathrm{CDF}$ is

$$
P_{R M}(A)=1-\sum_{i=1}^{m} \varepsilon_{i} e^{-A^{2} / \lambda_{R, i}}
$$

where

$$
\sum_{i=1}^{m} \varepsilon_{i}=1
$$

is required to ensure a valid CDF.

Although the component densities in a mixture distribution need not be Rayleigh, or even members of the same parametric family, ${ }^{40}$ Rayleigh-mixture distributions have been fit successfully to seafloor acoustic backscatter. ${ }^{7,24,27}$ Because mixture distributions have yet to be evaluated for reverberation from both boundaries and the volume, we shall test them below with data collected by the TVSS. We begin by describing the TVSS, the data, and aspects of the acoustic geometry that help in understanding the results.

\section{TVSS DATA}

\section{A. TVSS data collection}

The TVSS includes separate cylindrical projector and hydrophone arrays, with the same $0.53 \mathrm{~m}$ diameter, mounted coaxially on a cylindrical tow body. The projector array has 32 elements equally spaced $11.25^{\circ}$ apart around the cylinder and designed to produce a "toroidal" beam pattern that is meant to be omni-directional in the plane perpendicular to the cylinder's axis (usually across-track) and $3.7^{\circ}$ wide at -3 


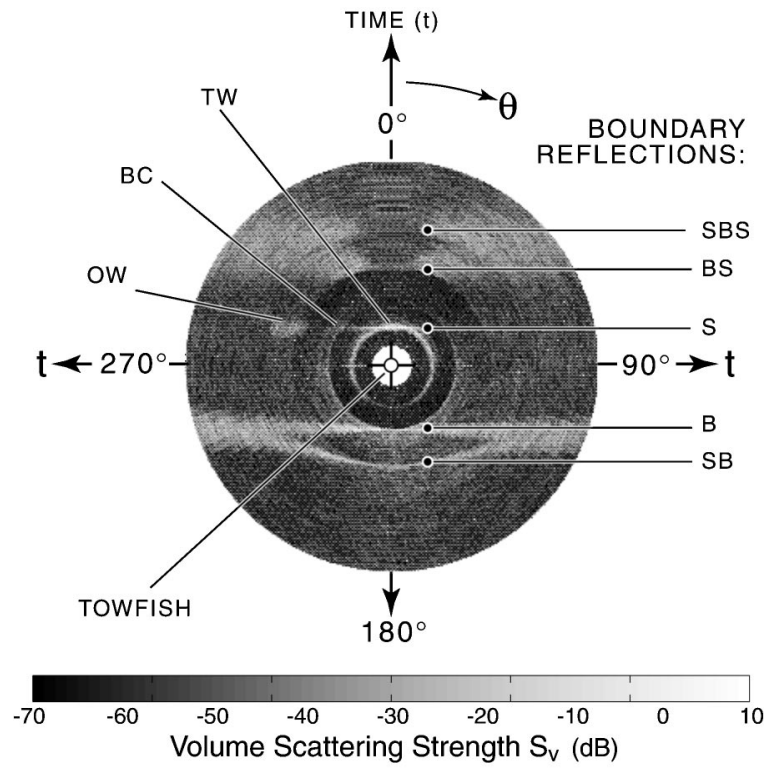

FIG. 3. Center locations of the analysis regions used in this study for a single TVSS ping. The data sets for each region consisted of 100 pings and spanned the horizontal and vertical dimensions listed in Table I.

$\mathrm{dB}$ in any plane containing the cylinder's axis (usually along-track). The hydrophone array consists of 120 elements equally spaced every $3^{\circ}$ around the cylinder. In the work presented here, split aperture beamforming of the hydrophone array yielded 120 receive beams, each $4.95^{\circ}$ wide at $-3 \mathrm{~dB}$ and spaced $3^{\circ}$ apart to cover the full $360^{\circ}$ around the array in the plane perpendicular to the array's axis. Details of the data processing are available in Refs. 12-14 and 41, 42.

The acoustic data were collected by the TVSS in a $2 \mathrm{~nm}^{2}$ area $65 \mathrm{~nm}$ southeast of Panama City, Florida, in the northeastern Gulf of Mexico. The TVSS was towed approximately $735 \mathrm{~m}$ aft of the towship MR. OFFSHORE at a nearly constant depth of $78 \mathrm{~m}$ (Fig. 1). Three runs of 100 consecutive pings of acoustic backscatter data, from $200 \mu$ s CW pulses of 68 $\mathrm{kHz}$ transmitted once a second, were obtained while the towship speed was nearly constant at $4.1 \mathrm{~m} / \mathrm{s}$. Towfish attitude and motion data were sampled at $1 \mathrm{~Hz}$ (once per ping) and included roll, roll rate, pitch, heading, speed, and depth. The environmental data collected during the experiment included a single CTD cast, which revealed the presence of an isothermal mixed layer with a temperature of $24.8^{\circ} \mathrm{C}$ extending to a depth of $49 \mathrm{~m}$, a thermocline between $49 \mathrm{~m}-150 \mathrm{~m}$ depth, and a nearly isothermal layer above the bottom with a temperature of $15.6^{\circ} \mathrm{C}$. The surface salinity was $35.1 \mathrm{ppt}$, and the surface sound speed was $1534 \mathrm{~m} / \mathrm{s}$. The wind speed recorded at 0658AM onboard Mr. OFFSHORE was 6 knots (3 $\mathrm{m} / \mathrm{s}$ ), and the sea state was 1.5 .

\section{B. TVSS acoustic geometry}

The statistical results are best interpreted with an understanding of the TVSS acoustic geometry, which may be obtained from Figs. 1-3. Figure 3 depicts a vertical slice of volume scattering strength $\left(S_{V}\right)$ perpendicular to the towfish axis, formed by displaying the acoustic data in each of the
120 TVSS receive beams in a single ping around the TVSS in coordinates of depth vs horizontal range. In this representation, echoes from the sea surface and seafloor appear as the high backscatter, horizontal features above and below the towfish. Scattering from resonant microbubbles in the towship's wake and from bubble clouds formed by breaking ship waves are responsible for the high backscattering strength features near the sea surface. The circular features result from boundary reflections received in the sidelobes of beams directed away from the boundary.

The angular sample spacing in this figure is the spacing between maximum response axes of adjacent beams: $\theta_{s}$ $=3^{\circ}$. The quadrature sampling time increment of $\tau_{s}$ $=160 \mu \mathrm{s}$ results in a $12 \mathrm{~cm}$ slant range sample spacing assuming a sound speed in seawater $c=1500 \mathrm{~m} / \mathrm{s}$. With the TVSS pulse length $\tau_{p}=200 \mu \mathrm{s}$, the bandwidth is $W$ $=0.88 / \tau_{p}=4.4 \mathrm{kHz}$, which yields a range resolution $\Delta R$ $=c / 2 W=17 \mathrm{~cm}$.

The volumetric resolution in each ping is determined by the spatial dimensions of the volume ensonified by the TVSS transmit pulse within each receive beam. We approximated the ensonified volumes $(V)$ in Table I as the ellipsoidal shell formed from the intersection of the transmitted pulse bound by the transmit beam pattern, and the receive beam. Thus, the dimensions of $V$ increase with slant range from the TVSS, and ensonified volumes at equal ranges from the TVSS in adjacent beams overlap by $39.4 \%$. The towfish's speed, $V_{\text {TVSS }}=4.1 \mathrm{~m} / \mathrm{s}$, and the relatively narrow fore-aft transmit beamwidth resulted in overlap between ensonified volumes in the same beam angle for consecutive pings, which increased with range beyond $62 \mathrm{~m}$.

On the boundaries, resolution is defined by the area $(A)$ ensonified by the transmitted pulse within each receive beam. The area is approximated by an ellipse near normal incidence, and by an annulus sector away from normal incidence. Thus, the maximum ensonified areas on the boundaries are at the towfish's zenith and nadir (Table I), where the horizontal resolution is poorest. Expressions for these and other characteristics of the TVSS acoustic geometry are given in Refs. 12-14.

\section{Data partitioning and description}

Applying sidescan imaging techniques to the TVSS data collected over multiple pings, we constructed seafloor, sea surface, and horizontal and vertical volume backscattering strength images, which are analyzed in Refs. 12-14. Four of these images are shown in Fig. 2 with their along-track averages. Whereas the seafloor image appears fairly homogeneous away from the track centerline [Fig. 2(a)], the sea surface and volume images exhibit significant spatial variability due to bubbles and bubble clouds [Figs. 2(b) and (c)] and aggregations of volume scatterers [Fig. 2(d)].

The acoustic backscatter amplitude data corresponding to these and other images were then partitioned into data sets which encompassed the analysis regions defined in Table I. The locations of the centers of these regions are indicated in Fig. 3. For 14 of the 15 analysis regions in Table I, three separate runs of 100 pings were used, and for one region 
TABLE I. Analysis regions for the TVSS data set. Negative across-track distances are left of the towfish's track. Grazing angles in regions VL1 and VL2 are defined with respect to the vertical along-track plane $47 \mathrm{~m}$ to the left of the towfish's track. Grazing angles in regions NS1-NS5 are defined with respect to the horizontal plane at $3 \mathrm{~m}$ depth. Ensonified areas (boundary regions SF, SS) and ensonified volumes (volume regions NS, VL) are listed in the last column.

\begin{tabular}{|c|c|c|c|c|c|}
\hline $\begin{array}{l}\text { Analysis } \\
\text { region }\end{array}$ & $\begin{array}{l}\text { Primary acoustic scattering and } \\
\text { reverberation features }\end{array}$ & $\begin{array}{l}\text { Across-track } \\
\text { distance(s) } \\
\quad(\mathrm{m})\end{array}$ & $\begin{array}{l}\operatorname{Depth}(\mathrm{s}) \\
(\mathrm{m})\end{array}$ & $\begin{array}{l}\text { Grazing } \\
\text { angles }\end{array}$ & $\begin{array}{l}\text { Areas or } \\
\text { volumes } \\
\left(\mathrm{m}^{2}, \mathrm{~m}^{3}\right)\end{array}$ \\
\hline SF1 & seafloor backscatter & $-35-+35$ & $192-202$ & $72^{\circ}-90^{\circ}$ & $4.5-73$ \\
\hline SF2 & seafloor backscatter + surface reverberation after first surface echo & $-50--100$ & $192-202$ & $48^{\circ}-66^{\circ}$ & $2.6-4.0$ \\
\hline SF3 & $\begin{array}{l}\text { seafloor backscatter }+ \text { surface and bottom reverberation after } \\
\text { surface-bottom multiple }\end{array}$ & $-150--200$ & $192-202$ & $29^{\circ}-37^{\circ}$ & $2.6-3.1$ \\
\hline SS1 & sea surface backscatter+attenuation from bubbles in towship's wake & $-30-+30$ & 0 & $68^{\circ}-90^{\circ}$ & $2-40$ \\
\hline SS2 & $\begin{array}{l}\text { sea surface backscatter+backscatter from bubble clouds generated } \\
\text { by ship and ambient waves }\end{array}$ & $40-80$ & 0 & $44^{\circ}-66^{\circ}$ & 2 \\
\hline SS3 & $\begin{array}{l}\text { sea surface acoustic backscatter+backscatter from bubble clouds } \\
\text { generated by ship and ambient waves + bottom reverberation from } \\
\text { first bottom echo }\end{array}$ & $100-150$ & 0 & $27^{\circ}-38^{\circ}$ & 2 \\
\hline NS1 & $\begin{array}{l}\text { near-surface volume and sea surface backscatter }+ \text { backscatter from } \\
\text { bubbles in towship's wake generated during previous runs }+ \text { surface } \\
\text { and bottom reverberation after bottom-surface multiple }\end{array}$ & $\begin{array}{l}180-220 \text { and } \\
-180--220\end{array}$ & 3 & $18^{\circ}-23^{\circ}$ & $37-52$ \\
\hline NS2 & $\begin{array}{l}\text { near-surface volume backscatter from bubbles within the towship's } \\
\text { wake+surface reverberation after first surface echo }\end{array}$ & $-30-+30$ & 3 & $68^{\circ}-90^{\circ}$ & $5-6$ \\
\hline NS3 & $\begin{array}{l}\text { near-surface volume and surface backscatter from bubble clouds } \\
\text { generated by ship waves }+ \text { surface reverberation after first surface } \\
\text { echo }\end{array}$ & $40-80$ & 3 & $42^{\circ}-62^{\circ}$ & $6-12$ \\
\hline NS4 & $\begin{array}{l}\text { near-surface volume backscatter from bubble clouds generated by } \\
\text { ship and ambient waves + surface and bottom reverberation after first } \\
\text { surface and bottom echoes }\end{array}$ & $100-150$ & 3 & $26^{\circ}-37^{\circ}$ & $15-27$ \\
\hline NS5 & surface and bottom reverberation after bottom-surface multiple & $\begin{array}{l}200-250 \text { and } \\
-200--250\end{array}$ & 3 & $16^{\circ}-20^{\circ}$ & $44-66$ \\
\hline VL1 & $\begin{array}{l}\text { volume backscatter from densely distributed zooplankton in mixed } \\
\text { layer and upper thermocline }\end{array}$ & -47 & $40-70$ & $50^{\circ}-81^{\circ}$ & $2-4$ \\
\hline VL2 & $\begin{array}{l}\text { volume backscatter from sparsely distributed zooplankton in middle } \\
\text { and lower thermocline }\end{array}$ & -47 & $90-120$ & $47^{\circ}-76^{\circ}$ & $2-4$ \\
\hline VL3 & $\begin{array}{l}\text { volume backscatter from sparsely distributed zooplankton in lower } \\
\text { thermocline }\end{array}$ & 0 & $125-140$ & $89^{\circ}-90^{\circ}$ & $2-4$ \\
\hline VL4 & $\begin{array}{l}\text { volume backscatter below thermocline from sparsely distributed fish } \\
+ \text { surface reverberation after first surface echo }\end{array}$ & 0 & $165-180$ & $89^{\circ}-90^{\circ}$ & $7-10$ \\
\hline
\end{tabular}

(NS1), two runs of 100 pings were used. Thus, the partitioning formed a total of 44 data sets.

Ideally, we would analyze the data collected in each grazing angle/depth/across-track distance location separately. However, this would have resulted in less than 100 samples per analysis region, and the PDF models and parameter estimation methods used here require much larger sample sizes to perform well. ${ }^{27}$ Therefore, we grouped data into the regions defined in Table I. To ensure that the data did not vary significantly over the range of grazing angles within each region, they were tested for homogeneity across both grazing angles and pings, as discussed below.

The partitioned data corresponding to the seafloor analysis regions span three different grazing angle regimes: normal and near normal incidence (SF1), moderate to high grazing angles (SF2), and moderate to low grazing angles (SF3). Bathymetry constructed from the TVSS backscatter data revealed a relatively flat bottom, with a $3 \mathrm{~m} / \mathrm{km}$ south west slope, and an average depth of $198 \mathrm{~m}$. Seafloor acoustic backscattering strength imagery indicated a homogeneous spatial distribution of sediments, and the angular dependence function estimated from the acoustic backscattering strength is consistent with the silt-sand mixture of sediments previ- ously surveyed in the region [e.g., Fig. 2(a)]. ${ }^{12}$

The sea surface analysis regions were influenced only slightly by sea surface roughness produced by the ambient 3 $\mathrm{m} / \mathrm{s}$ winds. Because of the vertical extent of the transmitted acoustic pulse intersecting the sea surface, the sea surface data were more strongly influenced by clouds of resonant mircobubbles which were characterized by different spatial dimensions and scattering characteristics that depended upon their generating mechanisms. These included (1) very dense bubble clouds generated primarily by propeller cavitation within the towship's wake (SS1), (2) large-scale $\left[\mathrm{O}\left(10^{2}\right)\right.$ to $\mathrm{O}\left(10^{3}\right) \mathrm{m}^{2}$ ] bubble clouds generated by breaking ship waves (SS2), and (3) sparsely distributed, small scale $[\mathrm{O}(1)$ to $\left.\mathrm{O}(10) \mathrm{m}^{2}\right]$ bubble clouds generated by the ambient sea (SS3) [e.g., Fig. 2(b)]. The SS3 region also was influenced strongly by bottom reverberation received in the sidelobes after the first bottom echo arrival. Although we did not have in-situ bubble size and density data, we used the resonant bubble approximation to estimate the densities of bubbles in the analysis regions from the surface and near-surface acoustic backscattering strength data in Ref. 13.

The near-surface volume regions were influenced by the same processes that influenced the sea surface backscatter. 
Clouds of resonant microbubbles in the towship's wake contributed to the backscatter in both the NS1 and NS2 regions, but these clouds were denser in NS1 than in NS2 because the wake in NS2 was about 20 minutes old, whereas the wake in NS1 was only 3 minutes old [Fig. 2(c)]. In the same acrosstrack location as the SS2 region, the NS3 region at $3 \mathrm{~m}$ depth was also affected by large scale bubble clouds generated by breaking towship waves. Similarly, the NS4 region was in the same across-track location as the SS3 region, and was also influenced by bottom reverberation and smaller scale bubble clouds generated by the ambient sea. The NS5 region was influenced by both near-surface bubbles and multiple boundary reflections occurring after the first bottom-surface multiple arrival. The NS1, NS3, NS4, and NS5 regions were influenced somewhat by surface roughness, due to the vertical extent of the ensonified volume.

Three of the volume regions were influenced by aggregations of zooplankton whose density generally decreased with depth from the base of the mixed layer (VL1), through the upper (VL2) and lower thermocline (VL3) [e.g., Fig. 2(d)]. ${ }^{14}$ The VL4 region near the bottom was influenced slightly by a sparse distribution of small fish, but more strongly by surface reverberation received in the sidelobes after the first surface echo arrival. As with the near-surface data, we lacked the in-situ data to characterize absolute densities and sizes of organisms in the volume, so we have inferred the relative densities from the corresponding volume acoustic backscattering strength data in Ref. 14. Although several dense fish schools were observed near the bottom, the backscatter data in these regions could not pass statistical independence tests, so they were not included in the analysis.

\section{Data preparation}

After grouping the TVSS acoustic backscatter amplitude data according to the analysis regions in Table I, data contaminated by noise spikes were removed. Because statistical analyses require independent and identically distributed data, the amplitudes were decimated by taking only those samples separated by at least a correlation width across grazing angles and pings. The correlation widths were estimated as the horizontal or vertical lags corresponding to the first null of the normalized spatial autocovariance. In cases where the autocovariance dropped sharply to a low value $(<0.1)$, and then fell gradually to zero, we used the distance for which it decreased to 0.1 .

As we are interested in reverberation fluctuations, we removed nonstationarities resulting from backscatter angular dependence and angular variations in the TVSS transmit and receive beam patterns by grouping the amplitude data in each analysis region into bins $1^{\circ}$ wide according to grazing angle and angle with respect to the TVSS, and then normalizing by the mean in each group. The normalized data were then regrouped into each analysis region (Table I), and inspected to ensure that all nonstationarities due to beam pattern variations and grazing angle dependence were adequately removed.

To ensure that the samples in each analysis region were statistically independent and identically distributed across pings and grazing angles, we performed the one sample runs test $^{43}$ for randomness and the Mann-Whitney $U$ test for homogeneity: ${ }^{44}(1)$ to the normalized samples in each grazing angle across pings, and (2) to the normalized samples in each ping across grazing angles. Most of the data in the seafloor (SF) and volume (VL) regions passed the tests at the $95 \%$ confidence level, but $20 \%-50 \%$ of the sea surface (SS) and near-surface (NS) data failed the tests. In studies of data collected on fixed platforms, the approach is to simply remove data which do not pass the tests at the specified confidence level. ${ }^{21}$ Doing so in our study was not possible because the TVSS data were collected from a moving platform. Therefore, for each analysis region, we selected only those samples within the largest contiguous regions (across pings and grazing angles) which passed both tests at the 95\% confidence level. We verified that the retained samples included contributions from the various backscattering and reverberation features in Table I by analyzing backscattering strength images formed from these data (e.g., Fig. 2).

\section{RESULTS}

\section{A. Backscattering strength, amplitude and intensity statistics}

The data in each of the analysis regions depicted in Fig. 3 were first characterized by averaging statistical estimates of the corresponding backscattering strength $\left(\mathrm{S}_{B, S, V}\right)$, amplitude $(A)$, and intensity $\left(A^{2}\right)$ over the three TVSS runs (Table II). Expressions for the mean $\left(\mu_{A}\right)$, variance $\left(\sigma_{A}^{2}\right)$, skewness $\left(\gamma_{3, A}\right)$, and kurtosis $\left(\gamma_{4, A}\right)$ are given in the Appendix. The scintillation index, which is the variance of the intensity fluctuation scaled by the square of the mean intensity, was computed as

$$
\sigma_{A^{2}}^{2}=\frac{\left\langle\left(A^{2}-\lambda_{R}\right)^{2}\right\rangle}{\lambda_{R}^{2}} .
$$

We include this quantity because it generally indicates the extent to which the data depart from a Rayleigh distribution, as Rayleigh-distributed amplitudes result in a scintillation index of one.

Table II shows that the scintillation indices for the seafloor regions are the closest to one, suggesting that they depart the least from Rayleigh distributions. In addition, the amplitude variance, skewness, and kurtosis values are lower for the seafloor regions. Mean backscattering strengths decrease away from the nadir region (SF1) [e.g., Fig. 2(a)], which is consistent with composite roughness model predictions for the silt-sand sediment type in the region and expected for rough-surface models of relatively smooth seafloors. ${ }^{12}$ The region at nadir also exhibits the highest variance, skewness, and kurtosis of the three seafloor regions.

Statistics for the sea surface regions differ significantly from those for the seafloor regions. The region at zenith (SS1) has the highest mean backscattering strengths of all regions, but these are attenuated approximately $22 \mathrm{~dB}$ below model predictions by resonant microbubbles in the towship's wake [e.g., Figs. 2(b) and (f)]. ${ }^{13}$ Backscattering strength decreases with grazing angle, but scintillation indices, skewness, and kurtosis increase with decreasing grazing angle. This trend is opposite that of the bubble densities inferred 
TABLE II. Average TVSS backscatter amplitude statistics. We calculated backscattering strengths using expressions in Refs. 12-14. The scintillation index is computed from Eq. (15) in the text. The range is the maximum minus the minimum amplitude, and all other terms are computed from expressions in the Appendix. Because they were computed from the normalized amplitudes, all quantities except the backscattering strength are dimensionless. The statistics have been averaged over runs 1-3, except those for the NS1 region, which were averaged over runs 2-3.

\begin{tabular}{|c|c|c|c|c|c|c|c|c|}
\hline $\begin{array}{l}\text { Analysis } \\
\text { region }\end{array}$ & $\begin{array}{c}\text { Mean } \\
\text { number of } \\
\text { samples } \\
N\end{array}$ & $\begin{array}{c}\text { Mean } \\
\text { backscattering } \\
\text { strength } \\
S_{B, S, V}(\mathrm{~dB})\end{array}$ & $\begin{array}{c}\text { Mean } \\
\text { normalized } \\
\text { amplitude } \\
\mu_{A}\end{array}$ & Range & $\begin{array}{c}\text { Variance } \\
\sigma_{A}^{2}\end{array}$ & $\begin{array}{c}\text { Skewness } \\
\gamma_{3, A}\end{array}$ & $\begin{array}{c}\text { Kurtosis } \\
\gamma_{4, A}\end{array}$ & $\begin{array}{c}\text { Scintillation } \\
\text { index } \\
\sigma_{A^{2}}^{2}\end{array}$ \\
\hline SF1 & 1505 & -19.4 & 1.0292 & 3.2242 & 0.3115 & 0.9022 & 1.4171 & 1.2262 \\
\hline SF2 & 3840 & -22.6 & 1.0037 & 3.1795 & 0.2794 & 0.6447 & 0.1927 & 1.0156 \\
\hline SF3 & 4406 & -28.7 & 0.9962 & 3.4766 & 0.2793 & 0.7185 & 0.4448 & 1.0646 \\
\hline SS1 & 1330 & -12.9 & 1.0108 & 4.4545 & 0.4469 & 1.1528 & 1.6478 & 1.8328 \\
\hline $\mathrm{SS} 2$ & 2725 & -41.5 & 0.9943 & 7.6032 & 0.4834 & 2.0793 & 10.329 & 3.6730 \\
\hline SS3 & 3951 & -51.3 & 0.9981 & 13.3277 & 0.3479 & 6.2665 & 119.56 & 14.358 \\
\hline NS1 & 2500 & -51.7 & 1.0604 & 3.5567 & 0.2902 & 0.9185 & 0.9124 & 1.0575 \\
\hline NS2 & 1823 & -28.4 & 1.0139 & 5.1983 & 0.3963 & 1.6726 & 4.7479 & 2.2058 \\
\hline NS3 & 2350 & -50.7 & 1.0159 & 8.0735 & 0.5233 & 2.4721 & 14.042 & 4.6218 \\
\hline NS4 & 4985 & -60.7 & 0.9977 & 9.0243 & 0.2773 & 3.6466 & 38.646 & 4.8148 \\
\hline NS5 & 7296 & -59.4 & 1.0010 & 9.2410 & 0.1785 & 4.2419 & 61.760 & 3.1274 \\
\hline VL1 & 2835 & -65.5 & 1.0001 & 3.8499 & 0.2687 & 1.0841 & 1.4969 & 1.0282 \\
\hline VL2 & 2522 & -73.4 & 1.0094 & 5.1744 & 0.5285 & 1.6516 & 3.0865 & 2.5656 \\
\hline VL3 & 2732 & -76.3 & 1.0015 & 5.3451 & 0.4954 & 2.3111 & 6.9617 & 3.2434 \\
\hline VL4 & 2591 & -72.2 & 1.0167 & 3.6934 & 0.2919 & 1.3342 & 2.3139 & 1.3993 \\
\hline
\end{tabular}

from backscattering strength imagery [Fig. 2(b)], ${ }^{13}$ as the highest densities occurr near the towfish zenith (SS1), lower densities occurr in the regions influenced by large scale bubble clouds produced by breaking ship waves (SS2), and the lowest bubble densities are observed farther across track (SS3) where the near-surface bubble population consists primarily of bubbles generated by small scale breaking of the ambient sea waves.

Statistics for three of the near-surface regions (NS2NS4) exhibit a grazing angle dependence similar to that of the sea surface regions (SS1-SS3), with mean backscattering strength decreasing, and skewness, kurtosis, and scintillation index increasing away from the towfish's zenith. For the NS1 and NS5 regions, backscattering strength increases with decreasing grazing angle as a result of the bottom-surface multiple echo. For the NS1 region, backscattering from microbubbles in the decaying towship's wake [Figs. 1 and 2(b)] also increases the mean volume backscattering strength above that for the higher grazing angle region (NS4). Although the NS1 and NS5 regions are at similar across-track locations and have the largest ensonified volumes of all the analysis regions (Table I), their statistics are dramatically different. The skewness, kurtosis, and the scintillation index values for the NS5 region are among the highest values of all the analysis regions, and result from sparsely distributed bubbles generated by small scale breaking of ambient sea waves. The corresponding values for the NS1 region are significantly lower, and are the result of scattering from the denser distribution of bubbles in the towship's wake from the previous run.

The statistics for the volume regions are similar to those for the near-surface and sea surface regions in that they are mostly influenced by the density of scatterers. For the VL1VL3 regions, mean backscattering strength decreases, and skewness, kurtosis, and scintillation index increase with depth, resulting from the decrease in zooplankton density with depth [e.g., Fig. 2(d)]. Statistics for the VL4 region depart from this trend, and this may be related to the influence of surface reverberation after the first surface echo.

Before fitting the various PDF models to the TVSS data, we evaluated their potential suitability for describing backscatter fluctuations by comparing plots of the skewness and kurtosis descriptors $\left(\beta_{1}, \beta_{2}\right)$ of the normalized backscatter amplitude data with the possible values for each PDF family (Fig. 4), where $\beta_{1}=\gamma_{3, A}^{2}$, and $\beta_{2}=\gamma_{4, A}+3$. The Appendix describes the basis for this figure, which is taken from Abraham, ${ }^{27}$ and Johnson et al. ${ }^{45}$ Although matching skewness and kurtosis does not imply that distributions are identical or even a good approximation to one another, estimates of $\beta_{1}$ and $\beta_{2}$ from data can provide an indication of which PDF families are appropriate to consider. Except for the lognormal distribution, all PDF models appear suitable for describing the seafloor backscatter data, which is closer to being Rayleigh distributed than the amplitude data in the other regions. Skewness and kurtosis descriptors estimated from the sea surface, near-surface, and volume backscatter amplitude data are spread among all the PDF models, but only the Rayleigh mixture model is flexible enough to encompass all the measurements.

\section{B. Probability distribution functions}

Rayleigh, $K$, Weibull, log-normal, and Rayleigh mixture distributions were fit to the empirical distribution functions corresponding to the backscatter amplitude data in each run and TVSS analysis region. Figures 5-8 show results of representative runs for the seafloor, sea surface, near-surface, and volume displayed as probabilities of false alarm (PFA $=1-\mathrm{CDF})$. PFA is the probability that the amplitude will be higher than or equal to a given value, and we use it to display 




FIG. 4. Plot of skewness descriptor $\left(\beta_{1}\right)$ vs kurtosis descriptor $\left(\beta_{1}\right)$ values [defined in the Appendix, Eqs. (A1) and (A2)] computed from the backscattered amplitude data collected in each run and analysis region in Table I. The Rayleigh distribution is represented by a point, whereas the $K$, Weibull, and log-normal distributions are represented by lines. The two-component Rayleigh mixture model is represented by the shaded region. The basis for this figure is presented in the Appendix

the results because it illustrates best the non-Rayleigh nature of the data, which is mostly seen in the tails of the distributions. Although backscatter amplitude is displayed in decibels on the abscissa of each plot, the distribution fits were obtained from the data in linear units.

We assessed the goodness of fit between the model distributions and the empirical distributions derived from the TVSS data with the nonparametric Kolmogorov-Smirnoff test statistic, defined as the maximum absolute difference between the theoretical $\operatorname{CDF}[P(A)]$ and that formed from the $N$ data samples: ${ }^{46}$

$$
D_{k s}=\max \left|P(A)-F_{N}(A)\right|, \quad-\infty<A<\infty .
$$

When the empirical data $\left[F_{N}(A)\right]$ are drawn from a population in which the random variable $A$ has a continuous distribution function $P(A)$, the limiting distribution of $D_{k s}$ derived by Kolmogorov is ${ }^{46}$

$$
\lim _{N \rightarrow \infty} P\left(D_{k s}<\frac{h}{\sqrt{N}}\right)=Q(h),
$$

where, for $h>0$,

$$
Q(h)=\sum_{i=-\infty}^{\infty}(-1)^{i} e^{-2 i^{2} h^{2}} .
$$

The KS value $p=(1-Q(h))$ represents the probability from 0 to 1 of observing a more extreme value of $D_{k s}$ under the null hypothesis that the data are distributed according to $P(A)$. The closer $p$ is to one, the more likely that the observed data follow the model CDF. Although this test is widely used to fit theoretical CDFs to empirical data, ${ }^{7,27} \mathrm{Eq}$. (18) is not strictly valid when parameters for the theoretical distribution are estimated from the empirical data. ${ }^{46}$ Therefore, we assessed also the relative goodness of fit for the different PDF models by computing the root mean square difference between the model and the empirical distribution functions

$$
D_{\mathrm{rms}}=\left[\frac{1}{N} \sum_{i=1}^{N}\left(P\left(A_{i}\right)-F_{N}\left(A_{i}\right)\right)^{2}\right]^{1 / 2}
$$

and averaged values over the three TVSS runs for each region (Table IV). In addition, rms differences were computed and averaged only for the samples in the distributions for which the PFA was less than $10^{-2}$ (Table V) in order to evaluate how well the model CDFs fit the TVSS data in the tails of the distributions. This "tail rms difference" was calculated because relatively high kurtosis values in the nearsurface and surface data suggested that large tails would be present in the distributions of these data (Table II).

Among all analysis regions and PDF model types, the seafloor amplitude data has the lowest rms differences and best statistical fits (KS $p$ values). All ranges of grazing angles (SF1-SF3) are non-Rayleigh, but the moderate grazing angle region (SF2) is relatively close to Rayleigh (Fig. 5). However, $K$, Weibull, and Rayleigh mixture distributions provide good fits to the distributions (Tables III and IV) and the tails (Table V). The Rayleigh mixture distributions show the best overall performance. In addition, rms differences and $\mathrm{KS} p$ values indicate that no significant advantage is gained by using more than three-components in the Rayleigh mixture. These results are generally consistent with those in Lyons and Abraham $^{7}$ for backscatter amplitude data from mud bottom types in the $40^{\circ}-60^{\circ}$ and $60^{\circ}-80^{\circ}$ grazing angle regimes. The KS $p$ values in Table III are slightly lower than theirs, probably because of spatial variations in the bottom. Such variations were not present in their data because they were collected from fixed platforms.

Backscatter amplitude fluctuations from the sea surface (Fig. 6) are more non-Rayleigh than those from the seafloor, and depend mostly upon grazing angle and the density of bubbles relative to the vertical extents of the ensonified volumes adjacent to the sea surface. For the zenith region (SS1), where very high densities of bubbles in the towship's wake attenuated the acoustic backscatter, only the Rayleigh mix- 

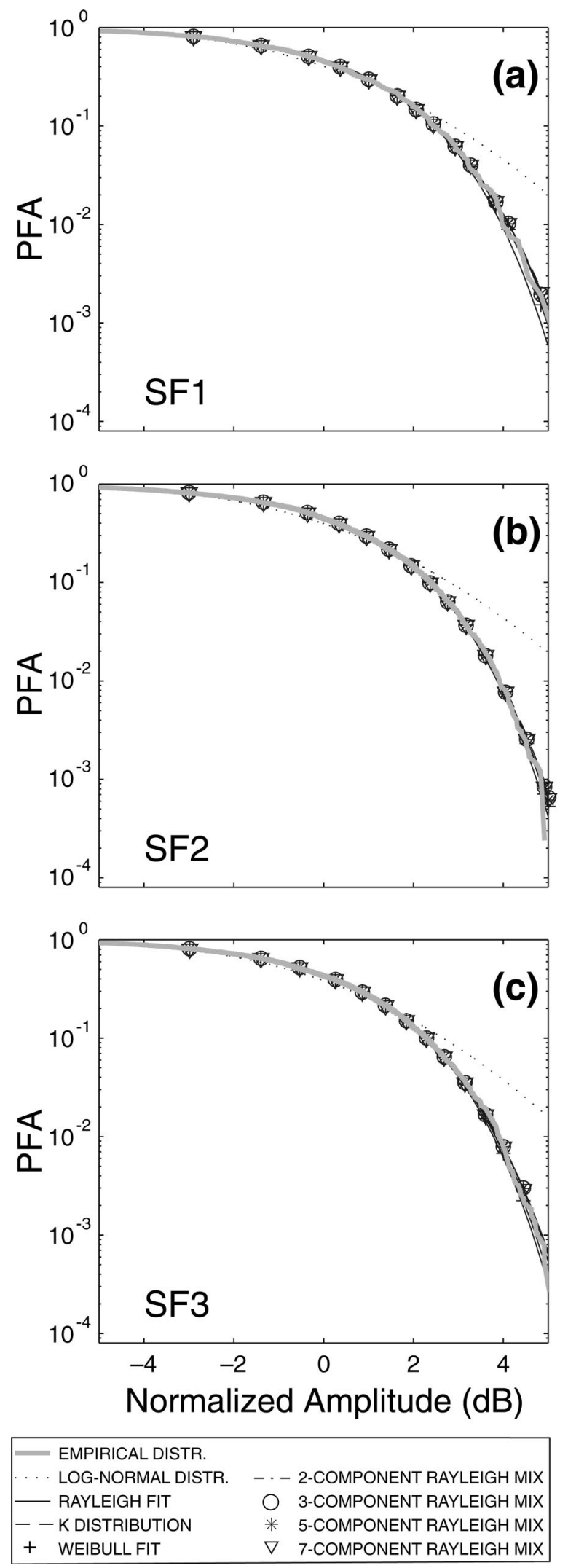

FIG. 5. Acoustic backscattered amplitude distributions displayed as probability of false alarm (PFA) for the three different seafloor regions in run 1 .

ture models provide statistically good fits to the observed data. Outside the wake, where bubble densities resulting from breaking waves generated by the towship were significantly lower than at zenith, KS $p$ values are lower and rms
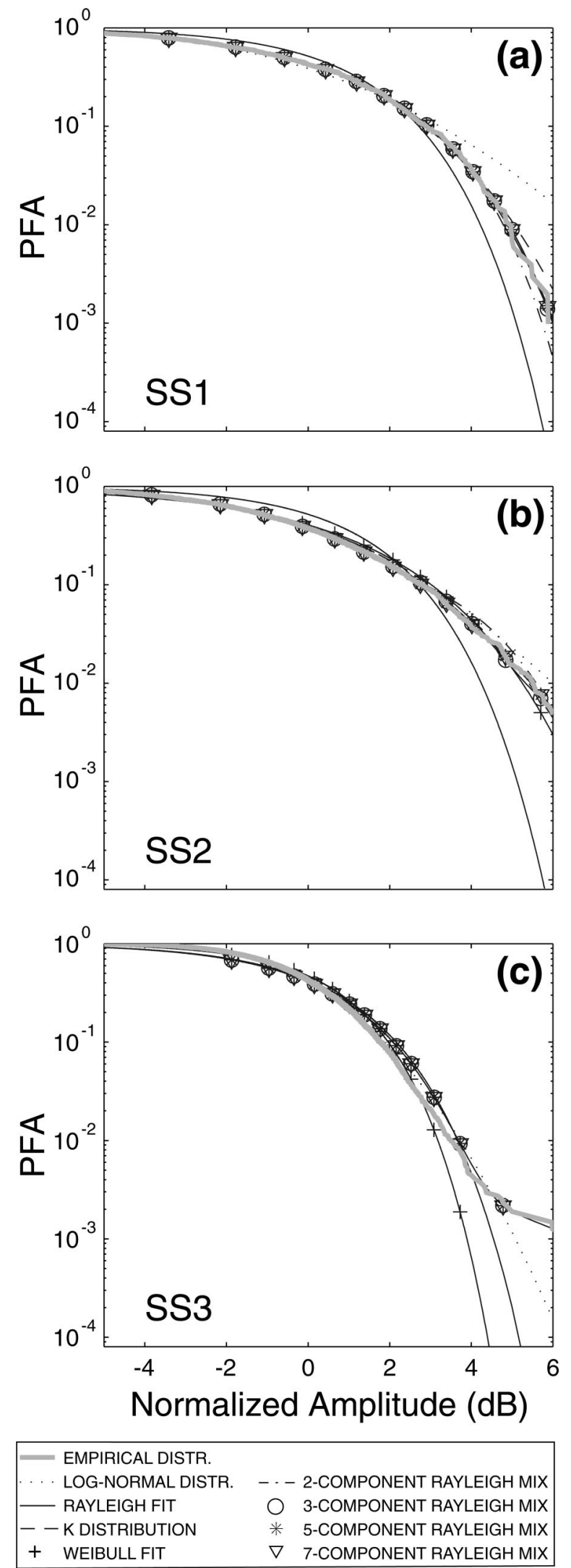

FIG. 6. PFA plots corresponding to the backscattered amplitude data from the sea surface regions in run 3 .

differences are higher, with Rayleigh mixture models again showing superior overall performance.

Backscatter amplitude distributions in the lowest grazing angle region $[\mathrm{SS} 3$, Fig. 6(c)] appear to be multimodal (cf. 

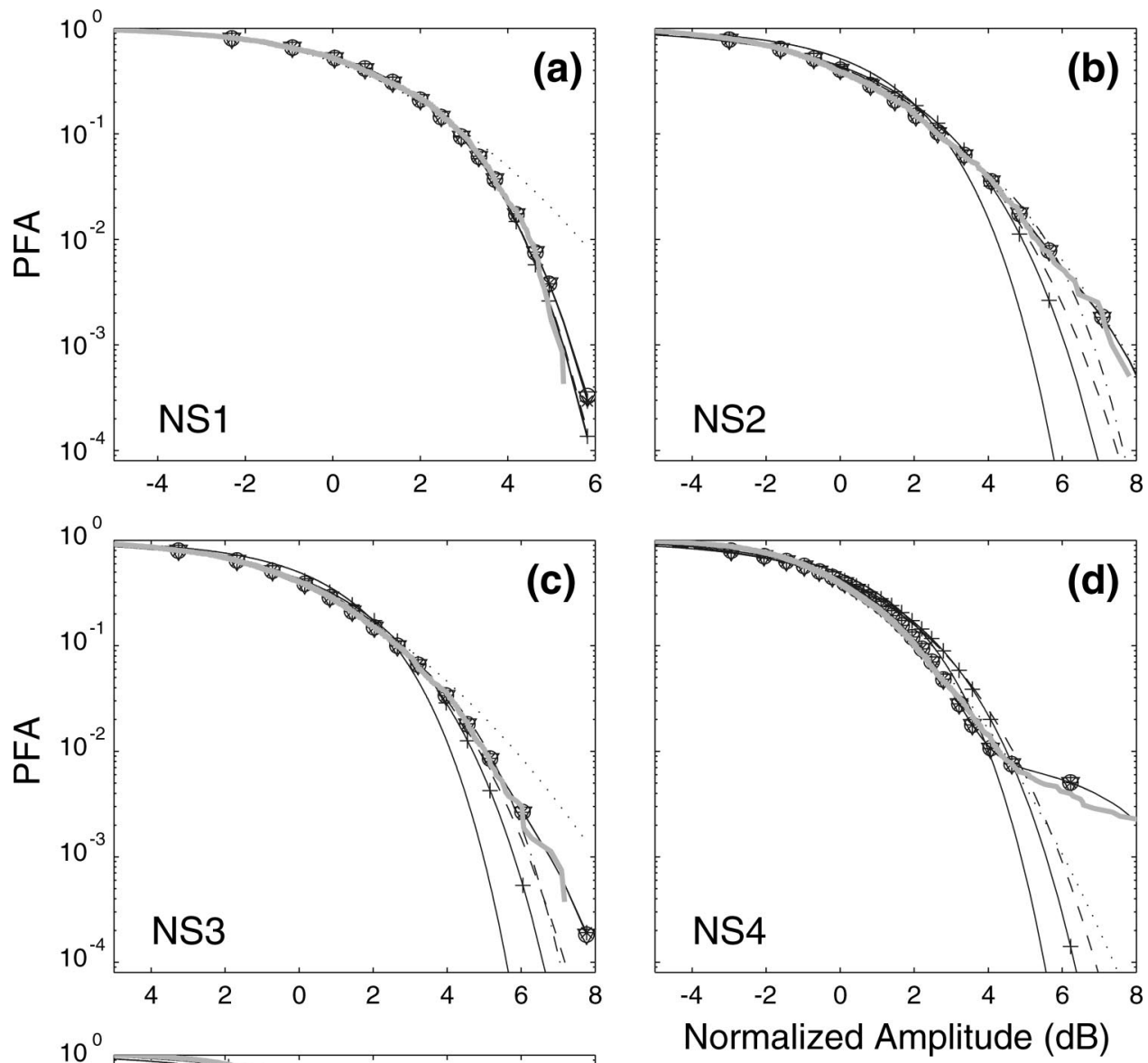

FIG. 7. PFA plots corresponding to the backscattered amplitude data from the near-surface regions in run 2 .
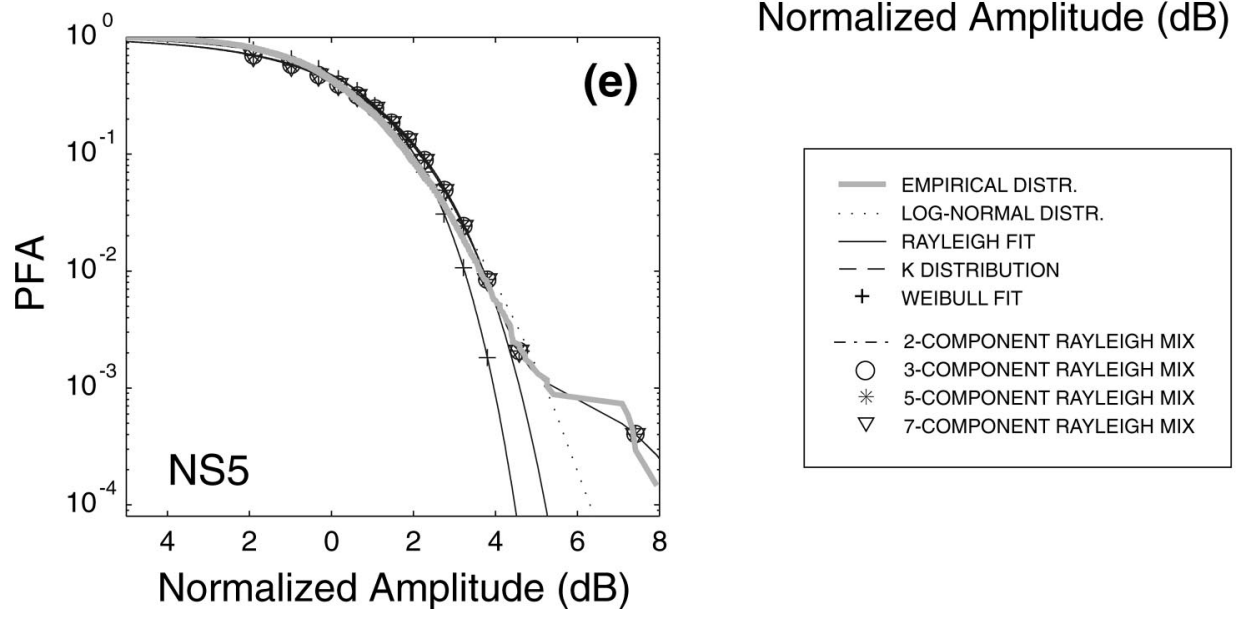

Ref. 40, Fig. 4.1.4). Analysis of Fig. 3 and the sea surface backscattering strength imagery corresponding to these data [e.g., Fig. 2(b)] indicates that the centers of the distributions for the SS3 regions are dominated by bottom reverberation, and the tails are dominated by randomly distributed bubbles within a meter of the sea surface. The log-normal model provides the best overall fits to the data in the SS3 regions (Tables III and IV) in terms of the KS $p$ value and rms difference which emphasize samples near the center of the distribution. As indicated by Fig. 6(c) and the rms differences for PFA values less than $10^{-2}$ (Table V), the Rayleigh mixture models provide the best fits to the tails in the SS3 regions.

Similar to the SS3 region, most of the near-surface data are best described by the log-normal model (Table III), but the Rayleigh mixture models provide the best fits to the tails
(Fig. 7; Table V). Results for the NS1 and NS3 regions are split, with the Rayleigh mixture and log-normal models both providing the best fits for different data runs (Table III). Overall, the model-data fits are statistically poor, and characterized by the highest rms differences and lowest KS $p$ values of all the analysis regions (Tables III and IV). Sidelobe returns from the bottom-surface multiple occur in the NS1 and NS5 regions, and sidelobe returns from the bottom echo are evident in the NS4 region. The outer edges of the NS1 region also are influenced by sidelobe returns from the first surface echo at the towfish's zenith, and the NS3 region is influenced by sidelobe returns from the first surface echo. The best fits for the log-normal distribution are in the regions where the boundary reverberation is the strongest (i.e., NS2, after the first surface echo; and NS4, after the first bottom echo). 

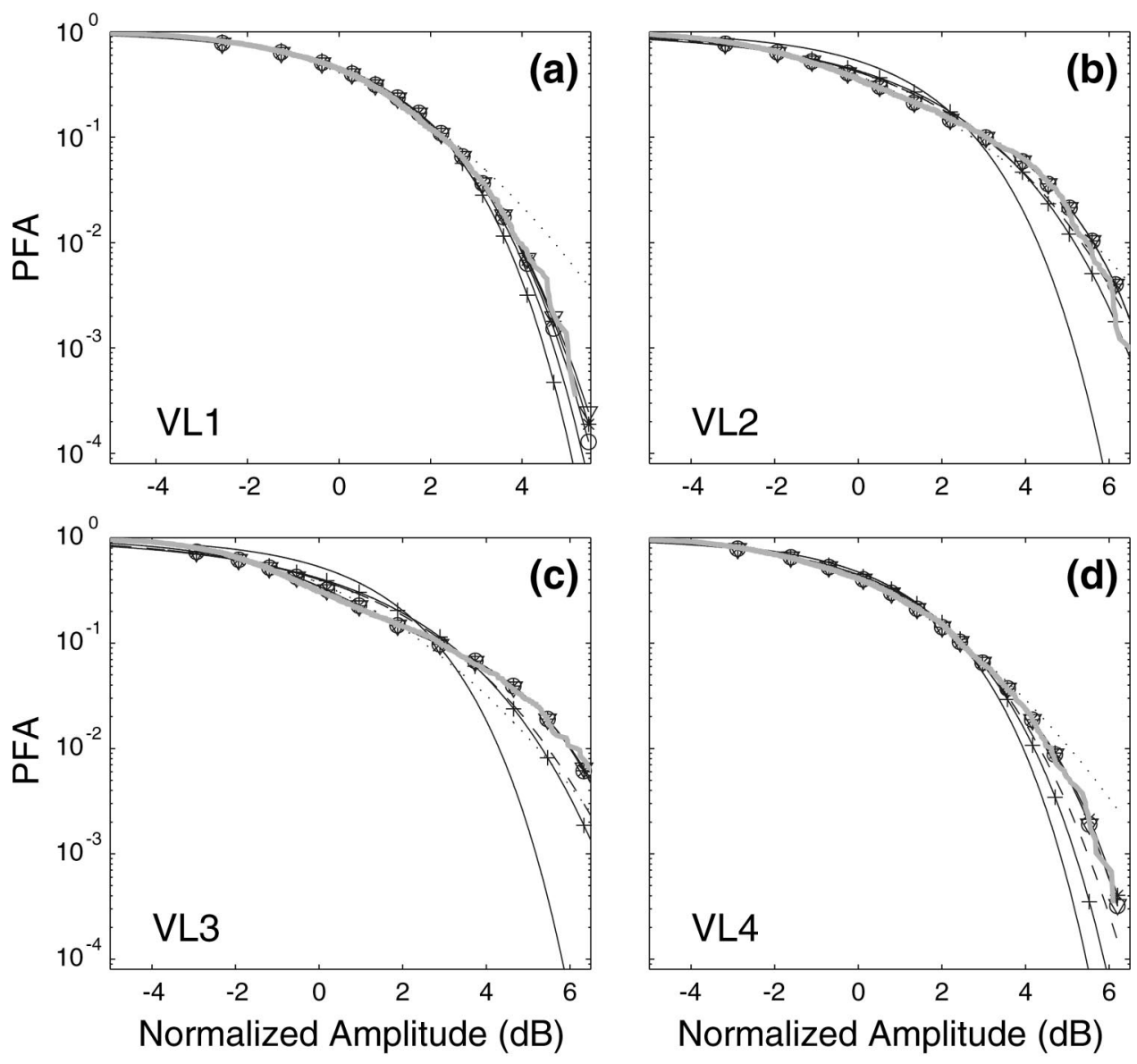

FIG. 8. PFA plots corresponding to the backscattered amplitude data from the volume regions in run 3.

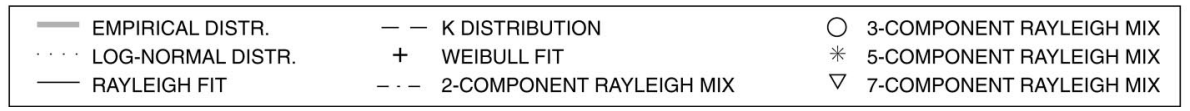

Analysis of backscattering strength imagery ${ }^{13}$ indicated that the dominant mechanisms contributing to the tails of the near-surface distributions are scattering from resonant microbubbles and bubble clouds, with bubble density controlling the tail shape. When the bubbles are sparsely distributed, such as those generated by the breaking ambient sea waves, the tail is well-separated from the distribution center, resulting in what appears to be a multi-modal distribution [NS4, Fig. 7(d); NS5, Fig. 7(c)]. When the bubbles are more densely packed, such as in the towship's wake (NS1,2) and in the region affected by large scale bubble clouds generated by ship-waves (NS3), the distributions appear unimodal, with the largest tails occurring in the regions with the highest bubble densities [NS2, Fig. 7(b)]. As with the sea surface results, little or no improvement in fitting the tails of the distributions occurred when we increased the number of components in the Rayleigh mixture above 3, and 2 components were sufficient in most cases.

Results for the volume backscatter amplitude data (Fig. 8) are generally similar to those for the near-surface data: they are best fit by the log-normal model over the center of the distribution and the Rayleigh mixture distributions in the tails. In addition, the fits are not statistically good, with relatively low KS $p$ values and high rms differences (Tables III-V). The best fits to the log-normal model are obtained when boundary reverberation is present, i.e., in data for the
VL4 region [Fig. 8(d)] that contain sidelobe returns after the first surface echo (Fig. 3). When boundary reverberation is absent, the shapes and tails of the distributions are affected by the density of the scatterers (zooplankton). For the highest scatterer densities [VL1, Fig. 8(a)], the distributions appear to be unimodal with lower tails. As the density of scatterers decreases, the distributions become multimodal, with heavier tails [VL2, Fig. 8(b); VL3, Fig. 8(c)]. These observations are somewhat consistent with those for the surface and nearsurface regions, in that a sparse, nonhomogeneous spatial distribution of scatterers (bubbles) results in distributions with more complex (multimodal) shapes [cf. SS3, Fig. 6(c); NS4, Fig. 7(d)].

\section{DISCUSSION}

\section{A. Nonstationarity of shallow water reverberation fluctuations}

Before offering physical arguments for the observed results, we address the fact that none of the regions could be considered stationary across all pings and grazing angles. The primary factors contributing to the observed nonstationarities are the towfish's motion through the generally nonhomogeneous spatial distribution of scatterers in each region, and boundary reverberation received in the sidelobes. This is evident in Table VI, which lists the samples sizes and percent 
TABLE III. KS statistic $p$-values computed from the model-data PDF fits to the TVSS acoustic backscatter amplitude data in each of the analysis regions. The highest value for each run is in bold and corresponds to the best fit.

\begin{tabular}{|c|c|c|c|c|c|c|c|c|}
\hline Region run/ & Rayleigh & $K$ & Weibull & Log-normal & $\begin{array}{l}\text { Rayleigh } \\
\text { 2-mixture }\end{array}$ & $\begin{array}{l}\text { Rayleigh } \\
\text { 3-mixture }\end{array}$ & $\begin{array}{l}\text { Rayleigh } \\
\text { 5-mixture }\end{array}$ & $\begin{array}{l}\text { Rayleigh } \\
\text { 7-mixture }\end{array}$ \\
\hline $\mathrm{SF} 1 / 01$ & 0.466 & 0.886 & 0.708 & $3.01 \times 10^{-4}$ & 0.936 & 0.932 & 0.936 & 0.940 \\
\hline$/ 02$ & 0.015 & 0.765 & 0.263 & $2.09 \times 10^{-4}$ & 0.890 & 0.890 & 0.982 & 0.984 \\
\hline$/ 03$ & 0.689 & & 0.928 & $9.25 \times 10^{-4}$ & 0.689 & 0.518 & 0.503 & 0.500 \\
\hline $\mathrm{SF} 2 / 01$ & 0.442 & 0.878 & 0.907 & $1.94 \times 10^{-21}$ & 0.443 & 0.901 & 0.939 & 0.939 \\
\hline$/ 02$ & 0.867 & 0.989 & 0.993 & $8.18 \times 10^{-17}$ & 0.869 & 0.907 & 0.890 & 0.878 \\
\hline$/ 03$ & 0.868 & & 0.913 & $6.02 \times 10^{-17}$ & 0.861 & 0.859 & 0.855 & 0.854 \\
\hline SF3/01 & 0.055 & 0.329 & 0.241 & $1.16 \times 10^{-13}$ & 0.568 & 0.520 & 0.489 & 0.541 \\
\hline$/ 02$ & 0.298 & 0.973 & 0.969 & $3.26 \times 10^{-15}$ & 0.987 & 0.978 & 0.967 & 0.960 \\
\hline$/ 03$ & 0.419 & 0.724 & 0.587 & $3.65 \times 10^{-25}$ & 0.432 & 0.769 & 0.424 & 0.788 \\
\hline $\mathrm{SS} 1 / 01$ & $2.19 \times 10^{-9}$ & 0.739 & 0.392 & 0.002 & 0.819 & 0.942 & 0.973 & 0.969 \\
\hline$/ 02$ & $1.05 \times 10^{-25}$ & 0.358 & 0.322 & $3.54 \times 10^{-5}$ & 0.267 & 0.999 & 0.999 & 0.999 \\
\hline$/ 03$ & $6.67 \times 10^{-13}$ & 0.666 & 0.764 & 0.013 & 0.901 & 0.998 & 0.998 & 0.998 \\
\hline $\mathrm{SS} 2 / 01$ & $2.18 \times 10^{-67}$ & $2.48 \times 10^{-4}$ & $7.41 \times 10^{-9}$ & $5.17 \times 10^{-4}$ & 0.131 & 0.840 & 0.975 & 0.983 \\
\hline$/ 02$ & $3.12 \times 10^{-16}$ & 0.395 & 0.011 & $2.69 \times 10^{-4}$ & 0.997 & 0.997 & 0.996 & 0.992 \\
\hline$/ 03$ & $5.81 \times 10^{-63}$ & $1.97 \times 10^{-7}$ & $7.27 \times 10^{-14}$ & 0.370 & 0.034 & 0.311 & 0.234 & 0.235 \\
\hline $\mathrm{SS} 3 / 01$ & $4.53 \times 10^{-23}$ & $3.14 \times 10^{-23}$ & $3.64 \times 10^{-29}$ & 0.342 & $7.53 \times 10^{-11}$ & $7.52 \times 10^{-11}$ & $7.44 \times 10^{-11}$ & $1.56 \times 10^{-12}$ \\
\hline$/ 02$ & $7.96 \times 10^{-60}$ & $2.36 \times 10^{-53}$ & $4.45 \times 10^{-70}$ & 0.260 & $7.28 \times 10^{-19}$ & $7.16 \times 10^{-19}$ & $1.33 \times 10^{-22}$ & $1.39 \times 10^{-22}$ \\
\hline$/ 03$ & $3.10 \times 10^{-64}$ & & $3.58 \times 10^{-14}$ & 0.045 & $5.21 \times 10^{-73}$ & $5.21 \times 10^{-73}$ & $5.21 \times 10^{-73}$ & $5.20 \times 10^{-73}$ \\
\hline NS1/02 & 0.031 & 0.043 & 0.038 & $1.13 \times 10^{-4}$ & 0.063 & 0.069 & 0.057 & 0.063 \\
\hline$/ 03$ & $3.03 \times 10^{-9}$ & & $2.58 \times 10^{-4}$ & 0.009 & $2.93 \times 10^{-9}$ & $6.57 \times 10^{-10}$ & $3.69 \times 10^{-10}$ & $3.13 \times 10^{-10}$ \\
\hline NS2/01 & $5.25 \times 10^{-10}$ & $1.40 \times 10^{-4}$ & $1.29 \times 10^{-5}$ & 0.113 & $1.43 \times 10^{-4}$ & $1.44 \times 10^{-4}$ & $1.44 \times 10^{-4}$ & $1.73 \times 10^{-4}$ \\
\hline$/ 02$ & $3.15 \times 10^{-32}$ & $2.21 \times 10^{-6}$ & $3.02 \times 10^{-11}$ & 0.649 & 0.016 & $6.12 \times 10^{-4}$ & $6.26 \times 10^{-4}$ & $6.26 \times 10^{-4}$ \\
\hline$/ 03$ & $7.97 \times 10^{-11}$ & 0.022 & $8.22 \times 10^{-4}$ & 0.433 & 0.083 & 0.081 & 0.082 & 0.079 \\
\hline NS3/01 & $5.65 \times 10^{-29}$ & 0.033 & $2.55 \times 10^{-5}$ & $1.95 \times 10^{-4}$ & 0.266 & 0.943 & 0.924 & 0.958 \\
\hline$/ 02$ & $5.29 \times 10^{-25}$ & 0.029 & $1.11 \times 10^{-5}$ & 0.004 & 0.485 & 0.632 & 0.627 & 0.631 \\
\hline$/ 03$ & $3.13 \times 10^{-53}$ & $2.37 \times 10^{-14}$ & $6.76 \times 10^{-21}$ & 0.277 & 0.031 & 0.100 & 0.093 & 0.088 \\
\hline NS4/01 & $2.63 \times 10^{-35}$ & & $5.64 \times 10^{-28}$ & 0.122 & $1.44 \times 10^{-49}$ & $1.43 \times 10^{-49}$ & $1.44 \times 10^{-49}$ & $1.43 \times 10^{-49}$ \\
\hline$/ 02$ & $1.01 \times 10^{-41}$ & $1.53 \times 10^{-44}$ & $1.07 \times 10^{-54}$ & 0.167 & $3.04 \times 10^{-25}$ & $3.03 \times 10^{-25}$ & $3.02 \times 10^{-25}$ & $2.92 \times 10^{-25}$ \\
\hline$/ 03$ & $1.22 \times 10^{-59}$ & & $5.57 \times 10^{-13}$ & 0.003 & $1.29 \times 10^{-70}$ & $1.29 \times 10^{-70}$ & $1.29 \times 10^{-70}$ & $1.29 \times 10^{-70}$ \\
\hline NS5/01 & $1.87 \times 10^{-91}$ & & $4.23 \times 10^{-30}$ & $1.03 \times 10^{-4}$ & $1.25 \times 10^{-108}$ & $1.24 \times 10^{-108}$ & $1.25 \times 10^{-108}$ & $1.25 \times 10^{-108}$ \\
\hline$/ 02$ & $3.29 \times 10^{-77}$ & & $2.17 \times 10^{-19}$ & $\mathbf{1 . 9 4} \times 10^{-4}$ & $1.86 \times 10^{-84}$ & $1.86 \times 10^{-84}$ & $1.86 \times 10^{-84}$ & $1.86 \times 10^{-84}$ \\
\hline$/ 03$ & $3.26 \times 10^{-145}$ & & $5.02 \times 10^{-13}$ & $7.58 \times 10^{-6}$ & $3.26 \times 10^{-145}$ & $3.26 \times 10^{-145}$ & $3.26 \times 10^{-145}$ & $3.26 \times 10^{-145}$ \\
\hline VL1/01 & $9.75 \times 10^{-8}$ & & $7.59 \times 10^{-6}$ & 0.023 & $3.59 \times 10^{-9}$ & $9.74 \times 10^{-8}$ & $4.21 \times 10^{-8}$ & $5.08 \times 10^{-9}$ \\
\hline$/ 02$ & $1.56 \times 10^{-10}$ & $6.55 \times 10^{-6}$ & $1.15 \times 10^{-7}$ & 0.303 & $1.21 \times 10^{-7}$ & $1.48 \times 10^{-7}$ & $1.79 \times 10^{-7}$ & $1.61 \times 10^{-9}$ \\
\hline$/ 03$ & $1.11 \times 10^{-7}$ & & $9.67 \times 10^{-4}$ & $\mathbf{5 . 8 8} \times 10^{-4}$ & $1.11 \times 10^{-7}$ & $1.61 \times 10^{-8}$ & $1.07 \times 10^{-8}$ & $9.76 \times 10^{-9}$ \\
\hline VL2/01 & $5.22 \times 10^{-68}$ & $3.44 \times 10^{-7}$ & $8.16 \times 10^{-14}$ & 0.029 & $7.16 \times 10^{-5}$ & $7.82 \times 10^{-5}$ & $6.76 \times 10^{-5}$ & $6.62 \times 10^{-5}$ \\
\hline$/ 02$ & $7.32 \times 10^{-89}$ & $6.25 \times 10^{-12}$ & $2.91 \times 10^{-19}$ & $2.20 \times 10^{-4}$ & $1.84 \times 10^{-5}$ & $1.88 \times 10^{-5}$ & $1.07 \times 10^{-5}$ & $9.06 \times 10^{-6}$ \\
\hline$/ 03$ & $2.20 \times 10^{-70}$ & $1.58 \times 10^{-14}$ & $1.05 \times 10^{-22}$ & $2.29 \times 10^{-4}$ & $1.28 \times 10^{-7}$ & $1.28 \times 10^{-7}$ & $1.37 \times 10^{-7}$ & $1.36 \times 10^{-7}$ \\
\hline VL3/01 & $1.41 \times 10^{-104}$ & $3.09 \times 10^{-28}$ & $2.98 \times 10^{-39}$ & $4.96 \times 10^{-11}$ & $1.36 \times 10^{-18}$ & $1.39 \times 10^{-18}$ & $1.52 \times 10^{-18}$ & $1.44 \times 10^{-18}$ \\
\hline$/ 02$ & $3.21 \times 10^{-26}$ & $1.14 \times 10^{-26}$ & $3.36 \times 10^{-35}$ & $2.04 \times 10^{-6}$ & $2.68 \times 10^{-25}$ & $2.68 \times 10^{-25}$ & $2.69 \times 10^{-25}$ & $2.71 \times 10^{-25}$ \\
\hline$/ 03$ & $4.20 \times 10^{-121}$ & $1.70 \times 10^{-38}$ & $2.65 \times 10^{-48}$ & $\mathbf{2 . 0 4} \times 10^{-11}$ & $3.87 \times 10^{-19}$ & $3.89 \times 10^{-19}$ & $4.32 \times 10^{-19}$ & $2.46 \times 10^{-19}$ \\
\hline VL4/01 & $8.27 \times 10^{-10}$ & & $2.66 \times 10^{-8}$ & 0.323 & $2.38 \times 10^{-13}$ & $2.40 \times 10^{-13}$ & $2.44 \times 10^{-13}$ & $2.86 \times 10^{-13}$ \\
\hline$/ 02$ & $4.30 \times 10^{-9}$ & & $3.01 \times 10^{-8}$ & 0.848 & $1.78 \times 10^{-14}$ & $1.78 \times 10^{-14}$ & $1.80 \times 10^{-14}$ & $1.86 \times 10^{-14}$ \\
\hline$/ 03$ & $1.13 \times 10^{-15}$ & $1.39 \times 10^{-7}$ & $9.09 \times 10^{-11}$ & 0.092 & $2.92 \times 10^{-8}$ & $2.98 \times 10^{-8}$ & $3.62 \times 10^{-8}$ & $3.00 \times 10^{-8}$ \\
\hline
\end{tabular}

of data in each analysis region that were validated across pings and grazing angles as stationary and homogeneous. The lowest percentages occur in regions where multiple reverberation components with widely varying characteristics are present. For example, the NS1 region has the smallest percentage of contiguous samples satisfying stationarity and is influenced by microbubbles within the decaying ship's wake, bubbles associated with breaking waves in the ambient sea, and multiple boundary reflections received in the sidelobes. Similarly, the NS3 region has a small percentage of contiguous samples satisfying stationarity, and it is influenced by large and small scale bubble clouds, sea surface backscatter, and sea surface reverberation in the sidelobes. On the other hand, the VL1 region has the largest percentage of contiguous samples satisfying stationarity, and it is influenced almost entirely by scattering from zooplankton.

Although the TVSS data set is unique in that it contains a wide variety of backscatter and reverberation processes received in narrow beams simultaneously, it is consistent with data in other studies which have observed that shallow water 
TABLE IV. RMS differences $\left(D_{\mathrm{rms}}\right)$ between model and TVSS-derived empirical CDFs averaged over runs $1-3$. The lowest value for each region is displayed in bold and corresponds to the model with the best overall fit to the empirical distribution function.

\begin{tabular}{ccccccccc}
\hline \hline $\begin{array}{c}\text { Analysis } \\
\text { region }\end{array}$ & Rayleigh & $K$ & Weibull & Log-normal & $\begin{array}{c}\text { Rayleigh } \\
\text { 2-mixture }\end{array}$ & $\begin{array}{c}\text { Rayleigh } \\
\text { 3-mixture }\end{array}$ & $\begin{array}{c}\text { Rayleigh } \\
\text { 5-mixture }\end{array}$ & $\begin{array}{c}\text { Rayleigh } \\
\text { 7-mixture }\end{array}$ \\
\hline SF1 & 0.0132 & 0.0070 & 0.0072 & 0.0359 & $\mathbf{0 . 0 0 6 0}$ & 0.0063 & 0.0062 & 0.0063 \\
SF2 & 0.0045 & $\mathbf{0 . 0 0 2 9}$ & 0.0032 & 0.0425 & 0.0045 & 0.0036 & 0.0034 & 0.0034 \\
SF3 & 0.0067 & 0.0041 & 0.0047 & 0.0395 & 0.0041 & $\mathbf{0 . 0 0 3 9}$ & $\mathbf{0 . 0 0 3 9}$ & $\mathbf{0 . 0 0 3 9}$ \\
& & & & & & & & \\
SS1 & 0.0694 & 0.0091 & 0.0102 & 0.0287 & 0.0074 & 0.0040 & $\mathbf{0 . 0 0 3 9}$ & 0.0040 \\
SS2 & 0.0836 & 0.0191 & 0.0300 & 0.0180 & 0.0086 & 0.0045 & $\mathbf{0 . 0 0 4 1}$ & $\mathbf{0 . 0 0 4 1}$ \\
SS3 & 0.0685 & 0.0649 & 0.0611 & $\mathbf{0 . 0 0 9 6}$ & 0.0490 & 0.0490 & 0.0490 & 0.0490 \\
NS1 & 0.0195 & 0.0155 & 0.0200 & $\mathbf{0 . 0 1 9 2}$ & 0.0213 & 0.0219 & 0.0219 & 0.0219 \\
NS2 & 0.0586 & 0.0246 & 0.0341 & $\mathbf{0 . 0 0 9 1}$ & 0.0154 & 0.0153 & 0.0153 & 0.0153 \\
NS3 & 0.0863 & 0.0282 & 0.0387 & 0.0164 & 0.0105 & $\mathbf{0 . 0 0 6 1}$ & $\mathbf{0 . 0 0 6 1}$ & $\mathbf{0 . 0 0 6 1}$ \\
NS4 & 0.0592 & 0.0601 & 0.0503 & $\mathbf{0 . 0 0 9 9}$ & 0.0542 & 0.0542 & 0.0542 & 0.0542 \\
NS5 & 0.0713 & & 0.0348 & $\mathbf{0 . 0 1 2 5}$ & 0.0744 & 0.0744 & 0.0744 & 0.0744 \\
VL1 & 0.0291 & 0.0265 & 0.0258 & $\mathbf{0 . 0 1 3 0}$ & 0.0237 & 0.0243 & 0.0240 & 0.0240 \\
VL2 & 0.1142 & 0.0376 & 0.0476 & $\mathbf{0 . 0 1 7 9}$ & 0.0203 & 0.0203 & 0.0203 & 0.0203 \\
VL3 & 0.1164 & 0.0670 & 0.0766 & $\mathbf{0 . 0 3 1 7}$ & 0.0459 & 0.0459 & 0.0459 & 0.0459 \\
VL4 & 0.0411 & 0.0258 & 0.0369 & $\mathbf{0 . 0 0 8 2}$ & 0.0322 & 0.0322 & 0.0322 & 0.0322 \\
\hline \hline
\end{tabular}

acoustic reverberation fluctuations received by moving and fixed platforms are often nonstationary. ${ }^{6,23}$ Consequently, our results regarding probability distribution functions pertain to locally stationary reverberation fluctuations embedded in larger scale, nonstationary processes.

\section{B. Suitability of Rayleigh mixture distributions}

The Rayleigh mixture distributions provide the best fits to the observed data in most of the boundary regions (SF1-3, SS1-2), and some of the near-surface regions (NS1,3). Thus, the Rayleigh mixture model adequately handles patchy, nonhomogeneously (non-Poisson) distributed scatterers. For example, the seafloor in the area of the TVSS experiment has a bimodal sediment composition of sand and silt, ${ }^{12}$ making the mixture of two Rayleigh random variables, hence a twocomponent Rayleigh mixture distribution a logical model for the observed backscatter amplitude distributions. Three, five, and seven component Rayleigh mixtures sometimes provided better fits probably because bottom roughness and the spatial variability of the bottom across each run introduced additional components.

For the sea surface and near-surface backscatter data in the SS1, SS2, and NS3 regions, bubble clouds with varying spatial scales are the most likely sources for the different components of the Rayleigh mixture distributions that were fit to the observed data. Previous analyses ${ }^{13}$ indicate that the bubble densities in the towship's wake (SS1) and in the bubble clouds generated by breaking ship waves (SS2,NS3) are relatively high, suggesting that the empirical distribution of backscatter in these regions could be modeled by a mixture of Rayleigh variables, each accounting for the different scales of the bubble clouds and scattering from the sea sur-

TABLE V. RMS differences ( $D_{\text {rms }}$ ) between model and TVSS-derived empirical CDFs for PFA values below $10^{-2}$, averaged over runs $1-3$. The lowest value for each region is displayed in bold and corresponds to the model with the best overall fit to the empirical distribution function.

\begin{tabular}{ccccccccc}
\hline \hline $\begin{array}{c}\text { Analysis } \\
\text { region }\end{array}$ & Rayleigh & $K$ & Weibull & Log-normal & $\begin{array}{c}\text { Rayleigh } \\
\text { 2-mixture }\end{array}$ & $\begin{array}{c}\text { Rayleigh } \\
\text { 3-mixture }\end{array}$ & $\begin{array}{c}\text { Rayleigh } \\
\text { 5-mixture }\end{array}$ & $\begin{array}{c}\text { Rayleigh } \\
\text { 7-mixture }\end{array}$ \\
\hline SF1 & 0.0020 & 0.0012 & 0.0015 & 0.0220 & 0.0011 & 0.0010 & $\mathbf{0 . 0 0 0 9}$ & $\mathbf{0 . 0 0 0 9}$ \\
SF2 & 0.0007 & 0.0006 & $\mathbf{0 . 0 0 0 5}$ & 0.0320 & 0.0007 & 0.0006 & 0.0006 & 0.0006 \\
SF3 & 0.0011 & $\mathbf{0 . 0 0 0 4}$ & 0.0007 & 0.0272 & $\mathbf{0 . 0 0 0 4}$ & 0.0006 & 0.0005 & 0.0005 \\
& & & & & & & & \\
SS1 & 0.0050 & 0.0018 & 0.0014 & 0.0200 & 0.0020 & 0.0014 & $\mathbf{0 . 0 0 1 3}$ & $\mathbf{0 . 0 0 1 3}$ \\
SS2 & 0.0054 & 0.0009 & 0.0024 & 0.0087 & 0.0007 & 0.0007 & $\mathbf{0 . 0 0 0 5}$ & $\mathbf{0 . 0 0 0 5}$ \\
SS3 & 0.0077 & 0.0043 & 0.0034 & 0.0015 & 0.0017 & 0.0017 & 0.0011 & $\mathbf{0 . 0 0 0 8}$ \\
& & & & & & & & \\
NS1 & $\mathbf{0 . 0 0 0 5}$ & $\mathbf{0 . 0 0 0 5}$ & 0.0007 & 0.0100 & 0.0009 & 0.0008 & 0.0007 & 0.0008 \\
NS2 & 0.0011 & 0.0006 & 0.0007 & 0.0031 & $\mathbf{0 . 0 0 0 6}$ & 0.0007 & $\mathbf{0 . 0 0 0 6}$ & 0.0007 \\
NS3 & 0.0010 & 0.0007 & 0.0009 & 0.0026 & 0.0006 & $\mathbf{0 . 0 0 0 2}$ & $\mathbf{0 . 0 0 0 2}$ & $\mathbf{0 . 0 0 0 2}$ \\
NS4 & 0.0011 & 0.0012 & 0.0011 & 0.0010 & $\mathbf{0 . 0 0 0 2}$ & $\mathbf{0 . 0 0 0 2}$ & $\mathbf{0 . 0 0 0 2}$ & $\mathbf{0 . 0 0 0 2}$ \\
NS5 & 0.0017 & & 0.0011 & $\mathbf{0 . 0 0 0 7}$ & 0.0009 & 0.0009 & 0.0009 & 0.0009 \\
& & & & & & & & \\
VL1 & 0.0027 & 0.0015 & 0.0030 & 0.0091 & 0.0013 & 0.0016 & 0.0011 & $\mathbf{0 . 0 0 1 0}$ \\
VL2 & 0.0055 & $\mathbf{0 . 0 0 1 0}$ & 0.0020 & 0.0048 & 0.0014 & 0.0013 & 0.0012 & 0.0013 \\
VL3 & 0.0057 & 0.0030 & 0.0041 & 0.0028 & 0.0020 & 0.0020 & 0.0020 & $\mathbf{0 . 0 0 1 9}$ \\
VL4 & 0.0046 & 0.0019 & 0.0041 & 0.00382 & $\mathbf{0 . 0 0 0 7}$ & 0.0008 & 0.0008 & 0.0008 \\
\hline \hline
\end{tabular}


TABLE VI. Sample sizes for the TVSS analysis regions. The total number of samples (column 2) corresponds to each region defined in Table I (column 1), averaged across the 3 runs. The validated samples (column 3) correspond to the data used to form the empirical distribution functions displayed in Figs. 5-8, and are the largest contiguous subsets of the regions validated as stationary and homogeneous across-pings and grazing angles.

\begin{tabular}{cccc}
\hline \hline $\begin{array}{c}\text { Analysis } \\
\text { region }\end{array}$ & $\begin{array}{c}\text { Total } \\
\text { number of } \\
\text { samples }\end{array}$ & $\begin{array}{c}\text { Number of } \\
\text { validated } \\
\text { samples }\end{array}$ & $\begin{array}{c}\text { Percent of } \\
\text { validated } \\
\text { samples }\end{array}$ \\
\hline SF1 & 2185 & 1505 & 69 \\
SF2 & 5529 & 3840 & 69 \\
SF3 & 9296 & 4406 & 47 \\
SS1 & 2616 & 1330 & 51 \\
SS2 & 6537 & 2725 & 42 \\
SS3 & 8374 & 3951 & 47 \\
NS1 & 10036 & 2500 & 25 \\
NS2 & 2829 & 1823 & 64 \\
NS3 & 6672 & 2350 & 35 \\
NS4 & 11383 & 4985 & 44 \\
NS5 & 12510 & 7296 & 58 \\
VL1 & 3412 & 2835 & 83 \\
VL2 & 3914 & 2522 & 64 \\
VL3 & 3974 & 2732 & 69 \\
VL4 & 4020 & 2591 & 64 \\
\hline \hline
\end{tabular}

face. This is supported by backscattering strength images constructed from the data in these regions [e.g., Fig. 2(b)] which reveal a patchy distribution of bubble clouds with varying length scales along and across track. ${ }^{13}$

Although the Rayleigh mixture models do not fit the observed near-surface and volume backscatter data well, they are effective in fitting the distribution tails for every type of analysis region (Table V). This is most noticeable for the regions in which bubbles contributed to the tails of the distributions [e.g., SS3, Fig. 6(d); NS2, Fig. 7(b); NS4], indicating that scattering from bubbles and bubble clouds could be described as a mixture of Rayleigh distributed random variables. These figures also suggest that acoustic backscatter and reverberation from these regions is probably best described by a mixture of both Rayleigh and non-Rayleigh random variables, and that the Rayleigh mixture model is flexible enough to describe that portion of the empirical distribution resulting from a mixture of Rayleigh random variables, even if the entire distribution does not fit such a model.

The flexibility of the Rayleigh mixture model comes from its unification of a number of physically based models. On one end, a Rayleigh mixture distribution dominated by a single component can approximate a single Rayleigh distribution, a Weibull distribution with $\beta=2$, and a $K$ distribution in the limit as $\nu$ tends to infinity when the scale $1 / \sqrt{\nu}$ is applied to the data. ${ }^{27}$ A Rayleigh mixture distribution can also approximate the $K$ distribution by quantizing the Rayleigh speckle and chi-distributed components, and equating the latter with the mixture proportions. ${ }^{7}$ This explains why the Rayleigh mixture model fits the data well when the Weibull or $K$ distributions do (e.g., SF2, Tables III and IV). In addition, Lyons and Abraham ${ }^{7}$ have related the Rayleigh mixture model to other models based on physical processes, such as Crowther's ${ }^{1}$ and McDaniel's ${ }^{3}$ for seafloor backscatter.

\section{Scattering processes approximated by log-normal distributions}

The log-normal distribution provides the best fits to most of the near-surface and volume backscatter data, but the fits are only good in the centers of the distributions where boundary reverberation in the sidelobes (NS1,2,4,5; VL4) or scattering from patchy aggregations of zooplankton (VL1,2,3) dominated the acoustic backscatter. Here, we offer several explanations for these results in terms of approximately log-normal distributions. Approximate results are sufficient because the model-data fits were never statistically good since KS $p$ values were always below 0.85 .

First, we consider the near surface and volume data in which boundary reverberation in the sidelobes dominated the distribution centers (NS1,2,4,5,VL4). Because the amplitude data are validated as stationary, independent, and identically distributed across-grazing angles and pings, we can use the model in (1) and (2) to express the corresponding in-phase and quadrature components as ${ }^{16}$

$$
V_{I, Q}(r)=\frac{1}{g(r)} \sum_{i=1}^{n} a_{i} B\left(r_{i}\right) s\left(r-r_{i}, \xi_{i}\right)
$$

where the generalized variable $r$ is used in place of $t$ to represent the ranges of grazing angle, depth, along-track, or across-track distance in Table I. For the TVSS data, $g(r)$ represents the normalization procedure. Equation (20) can be rewritten as

$$
V_{I, Q}(r)=\sum_{i=1}^{n} \nu_{i}(r)
$$

where $\nu_{i}(r)$ is the reverberation component in the sum of (20) made stationary by $g(r)$. If we assume that each $\nu_{i}(r)$ can be expressed in terms of a random proportion of the preceding term $\nu_{i-1}(r)$, then

$$
\nu_{i}(r)=\nu_{i-1}(r)+\zeta_{i} \nu_{i-1}(r)
$$

where the random set $\left\{\zeta_{i}\right\}$ is mutually independent and independent of the set $\left\{\nu_{i}(r)\right\}$. This assumption is reasonable if the scattered amplitudes $a_{i}$ and stochastic parameters $\xi_{i}$ are random, as assumed in (1) and (20). Rearranging (22), we have

$$
\frac{\nu_{i}(r)-\nu_{i-1}(r)}{\nu_{i-1}(r)}=\zeta_{i}
$$

so that

$$
\sum_{i=1}^{n} \frac{\nu_{i}(r)-\nu_{i-1}(r)}{\nu_{i-1}(r)}=\sum_{i=1}^{n} \zeta_{i} .
$$

Now, supposing the difference between successive reverberation components $\nu_{i}(r)$ is small,

$$
\sum_{i=1}^{n} \frac{\nu_{i}(r)-\nu_{i-1}(r)}{\nu_{i-1}(r)} \approx \int_{\nu_{1}(r)}^{\nu_{n}(r)} \frac{d \nu}{\nu}=\ln \left(\nu_{n}(r)\right)-\ln \left(\nu_{1}(r)\right),
$$


which, from (24), becomes

$$
\ln \left(\nu_{n}(r)\right)=\ln \left(\nu_{1}(r)\right)+\zeta_{1}+\cdots+\zeta_{n} .
$$

For large $n$, the central limit theorem implies that $\ln \left(\nu_{n}(r)\right)$ is normally distributed, so $\nu_{n}(r)$ is log-normally distributed by the properties of the log-normal distribution discussed in Sec. II. If the minimum number for which (26) converges to log-normal is much less than the total number of scatterers $(n)$, then $V_{I, Q}(r)$ in Eq. (20) will be approximately log-normal because the sum of log-normal variables is approximately log-normal. ${ }^{38}$ By the same virtue, the TVSS amplitude data that are related to the in-phase and quadrature components by

$$
A(r)=\sqrt{V_{I}^{2}(r)+V_{Q}^{2}(r)}
$$

are approximately log-normal because a log-normal variable raised to a power is also log-normally distributed. ${ }^{39}$

From this development one might wonder why Eq. (26) converges to a normal distribution, but not Eq. (20). The central limit theorem may be invoked for Eq. (20) when $n$ is large, but the value of $n$ for which Eq. (20) converges to a normal distribution will be smaller if the variables $\nu_{i}(t)$ are from the same underlying distributions. This can be expected when the total scattered signal arises from numerous scatterers of the same type. The value of $n$ for which Eq. (20) converges to a normal distribution will be larger when each $\nu_{i}(r)$ arises from a different underlying distribution, especially those which are highly skewed or have large tails. ${ }^{44,47}$ This is likely to occur for volume backscatter received by a moving platform and dominated by boundary sidelobe returns, because each sidelobe is directed towards a different grazing angle with respect to the boundary (see Ref. 41 for the receive beam patterns of the TVSS). The total reverberation will be the sum of the components arriving in each sidelobe, where each component follows a different parent distribution. Thus, a log-normal distribution may approximate the observed data better than a normal distribution when the underlying distributions for the proportions $\zeta_{i}$ are less skewed, with smaller tails, than those for $\nu_{i}(r)$.

Perhaps the log-normal model best fits the volume backscatter data in and above the thermocline (VL1,2,3) because several biological and physical factors affecting the distribution of zooplankton are log-normally distributed. In observations made by Dugan et al. ${ }^{48}$ the distribution of horizontal temperature fluctuations in the seasonal thermocline followed a log-normal distribution on scales from $10 \mathrm{~cm}$ to 1 $\mathrm{km}$, whereas Campell's ${ }^{49}$ analysis showed that a variety of factors related to phytoplankton, such as chlorophyll concentration and cell size, are log-normally distributed. The spatial distribution of zooplankton in the northeastern Gulf of Mexico can be related to both of these. Zooplankton have been shown to be concentrated near the mixed layer depth, which is influenced by both weather and mixing processes, and also near the depth of the primary productivity maximum, which is related to phytoplankton, hence chlorophyll, distribution. ${ }^{14}$ Because these factors influence the number $n$ of scatterers in (20), they directly influence the empirical distributions of the backscatter amplitude fluctuations.
It is interesting to note that the log-normal distribution has been used to describe a wide variety of physical phenomena which may be indirectly related to volume acoustic backscatter fluctuations. In theoretical biology, for example, species abundance has been successfully described by the log-normal model, and organism growth was the first application that used the development in Eqs. (20)-(26), which is known as the law of proportional effect. ${ }^{50}$ The TVSS volume backscatter fluctuations are influenced by the size and species abundance distribution of sound scattering zooplankton through their backscattering cross sections, which are represented by the $a_{i}$ terms in (20). Although backscattering strength imagery indicated that the spatial distribution of zooplankton was nonhomogeneous [e.g., Fig. 2(d)], we did not collect net tow or trawl samples to verify whether the approximately log-normal distributions of the volume backscatter fluctuations were related to log-normal size or species abundance distributions of volume scatterers. Nevertheless, such observations are not likely to be purely coincidental, and deserve further investigation, in view of other studies in which high frequency volume acoustic backscatter from biological sound scatterers was approximately lognormal. $^{29,36,51}$

A final observation for approximately log-normal acoustic backscatter and reverberation distributions is that a lognormal distribution can be expressed as a mixture of several physically relevant distributions. Although we do not think this is the reason for our observations, this might explain others' (e.g., Refs. 35 and 23). For example, Titterington et $a l .{ }^{40}$ show an example in which two Gaussian distributions are used to approximate a two-parameter log-normal distribution. ${ }^{40}$ Thus, two Ricean distributed scattering processes, each dominated by a coherent scattering component, might yield a distribution which is approximately lognormal. Similarly, the log-normal distribution may be closely approximated by the gamma distribution, ${ }^{52}$ which has been directly related to a variety of scattering processes by Middleton. ${ }^{53}$ This is appropriate for shallow water reverberation, which typically includes multiple components from the boundaries and volume. These considerations are consistent with our previous conclusions that mixture distributions with component densities from different parametric families may better describe fluctuations of acoustic backscatter and reverberation in shallow water.

\section{Implications for target detection}

This study has several implications for undersea target detection. The non-Rayleigh nature of envelope fluctuations that arise from nonhomogeneous spatial distributions of scatterers is seen in large tails and/or multimodality in the distributions. For a predetermined probability of false alarm (PFA), this implies that threshold detectors which assume Rayleigh-distributed envelope fluctuations will experience significantly higher false alarm rates. However, even with the appropriate PDF model for envelope fluctuations due to the environment, target detection is difficult for data within the tails of the distributions. For these data, combining statistical 
techniques with analyses of multibeam imagery, as we have done here, may be a more effective method for discriminating between targets and noise.

\section{SUMMARY AND CONCLUSIONS}

In this study, we have analyzed the fluctuations of seafloor, sea surface, and volume acoustic backscatter data simultaneously collected by the Toroidal Volume Search Sonar (TVSS) while it was towed in a shallow water region in the northeastern Gulf of Mexico. The $68 \mathrm{kHz}$ acoustic backscatter data were grouped according to 15 analysis regions in which scattering contributions from the volume and/or boundaries were present. After normalizing for backscatter variations due to grazing angle dependence and nonuniformity in the TVSS's beam patterns, the data were validated as independent and identically distributed. Various moments and statistics were estimated for the data in each region, and Rayleigh, $K$, Weibull, log-normal, and Rayleigh mixture probability distributions were fit to the empirical distribution functions in each region. We used previously published volume and boundary acoustic backscattering strength images constructed from the multibeam data collected by the TVSS to interpret the observed backscatter and reverberation statistics in terms of the spatial distribution of scatterers.

Rayleigh mixture models provided the best fits to the backscatter data collected from both boundaries, and in most cases, three-component mixtures adequately described the observed data. For the near-surface and volume regions, none of the models considered yielded statistically good fits. The Rayleigh mixture distributions provided the best fits to the larger tails in the data for these regions, which were mostly due to sparse distributions of bubbles near the surface or zooplankton in the mixed layer and thermocline. The lognormal distribution best fit the centers of the distributions for the near-surface and volume regions, particularly when single and multiple boundary interactions were received in the sidelobes. Together, these observations suggest that mixture distributions with component densities from different parametric families might better describe the multiplecomponent reverberation that is typical of most shallow water environments.

With mixture distributions, it is difficult to determine the number of components required to represent the data, or what PDF families are appropriate, particularly since several different mixtures can be used to approximate the same distribution. This guess work can be reduced by identifying candidate mixture components based on the spatial distribution of scattering features observed in coincident acoustic backscatter imagery.

The results were displayed as probabilities of false alarm (PFAs) in order to emphasize the larger tails of the nonRayleigh backscatter statistics. The tails corresponding to data in the near-surface, sea-surface, and volume were much larger than those for the seafloor. Large tails resulted mostly from nonhomogeneous spatial distributions of bubbles near the sea surface, and zooplankton and small fish at the base of the mixed layer and in the thermocline. Multimodal distributions with extended tails were observed when the data were influenced by both discrete scatterers and multiple boundary interactions received in the sidelobes. The results demonstrate that the dominant environmental sources of noise in shallow water target detection applications are likely to be resonant microbubbles near the surface, aggregations of zooplankton and fish in the mixed layer and upper thermocline, and boundary reverberation throughout the entire water column.

\section{ACKNOWLEDGMENTS}

This work was funded by the Office of Naval Research under ONR-NRL Contract No. N00014-96-1-G913. The authors would like to thank CAPT. Tim Schnoor, USN (ret) (ONR), Sam Tooma, and Maria Kalcic (NRL) for their support; Candy Robertson and Lisa Tubridy (CSS) for information on the TVSS; and Pat Jordan (MPL) for administrative support. Special thanks are due to Doug Abraham for providing Ref. 27, which was essential in completing this work. Thanks are due to Jo Griffith (MPL) for helping with the figures.

\section{APPENDIX: SKEWNESS AND KURTOSIS DESCRIPTORS}

Expressions for the skewness and kurtosis descriptors in Fig. 4 are given by

$$
\beta_{1}=\gamma_{3, A}^{2}, \quad \beta_{2}=\gamma_{4, A}+3,
$$

which are obtained from the moments

$$
\begin{aligned}
& \mu_{A}=E[A] \quad(\text { mean }), \\
& \sigma_{A}^{2}=E\left[\left(A-\mu_{A}\right)^{2}\right] \quad \text { (variance), } \\
& \gamma_{3, A}=\frac{E\left[\left(A-\mu_{A}\right)^{3}\right]}{\sigma_{A}^{3}} \quad \text { (skewness), } \\
& \gamma_{4, A}=\frac{E\left[\left(A-\mu_{A}\right)^{4}\right]}{\sigma_{A}^{4}} \quad \text { (kurtosis). }
\end{aligned}
$$

Skewness and kurtosis are measures of departure from normality. Skewness represents asymmetry in the PDF, and high kurtosis indicates a relatively large number of values near the mean of the distribution.

For the TVSS data, the sample moments were calculated using (A2) with

$$
E[u]=\frac{1}{N} \sum_{i=1}^{N} u_{i}
$$

for the expected value.

For the PDF models, expressions for $\beta_{1}$ and $\beta_{2}$ in terms of the noncentral moments

$$
\alpha_{i}=E\left[A^{k}\right]=\int_{-\infty}^{\infty} A^{k} p(A) d A,
$$

where $p(A)$ is the probability density function of $A$, can be obtained by using the $k$ central moments

$$
E\left[\left(A-\mu_{A}\right)^{k}\right]=\int_{-\infty}^{\infty}\left(A-\mu_{A}\right)^{k} p(A) d A,
$$

yielding 27,54 


$$
\beta_{1}=\frac{\left(\alpha_{3}-3 \alpha_{1} \alpha_{2}+2 \alpha_{1}^{3}\right)^{2}}{\left(\alpha_{2}-\alpha_{1}^{2}\right)^{3}}
$$

and

$$
\beta_{2}=\frac{\alpha_{4}-4 \alpha_{1} \alpha_{3}+6 \alpha_{1}^{2} \alpha_{2}-3 \alpha_{1}^{4}}{\left(\alpha_{2}-\alpha_{1}^{2}\right)^{2}} .
$$

From these expressions and knowledge of the noncentral moments of the various PDF models, one can obtain the skewness and kurtosis descriptors plotted in Fig. 4. The $k$ th noncentral moment of the Rayleigh distribution [Eq. (3)] is ${ }^{45}$

$$
E\left[A^{k}\right]=\lambda_{R}^{k / 2} \Gamma\left(1+\frac{k}{2}\right),
$$

which can be used with (A6) and (A7) to show that it is represented by a single point in the $\left(\beta_{1}, \beta_{2}\right)$ plane

$$
\beta_{1}=\frac{4 \pi(\pi-3)^{2}}{(4-\pi)^{3}}
$$

and

$$
\beta_{2}=\frac{32-3 \pi^{2}}{(4-\pi)^{2}},
$$

because the Rayleigh distribution is fully represented by a single scale parameter $\left(\lambda_{R}\right)$, and skewness and kurtosis are scale invariant descriptors.

One can use the $k$ th noncentral moments of the twoparameter $K$, Weibull, and log-normal distributions to show that each of these is represented by a line in the $\left(\beta_{1}, \beta_{2}\right)$ plane (Fig. 4). The noncentral moments of the $K$ distribution [Eq. (5)] $\operatorname{are}^{27}$

$$
E\left[A^{k}\right]=\alpha^{k / 2} \Gamma\left(1+\frac{k}{2}\right) \frac{\Gamma\left(\nu+\frac{k}{2}\right)}{\Gamma(\nu)},
$$

those for the Weibull distribution [Eq. (7)] $\operatorname{are}^{45}$

$$
E\left[A^{k}\right]=\alpha^{-k / \beta} \Gamma\left(1+\frac{k}{\beta}\right),
$$

and the moments of the log-normal distribution [Eq. (9)] $\operatorname{are}^{39}$

$$
E\left[A^{k}\right]=e^{\left(k \beta+(1 / 2) k^{2} \alpha^{2}\right)},
$$

where $\alpha, \beta$, and $\nu$ are the parameters of the various distributions as defined in the text.

The $m$-component Rayleigh mixture distribution [Eq. (12)] is represented by a region in the $\left(\beta_{1}, \beta_{2}\right)$ plane, which can be seen by using the equation for the $k$ th noncentral moment: ${ }^{27}$

$$
E\left[A^{k}\right]=\sum_{i=1}^{m} \varepsilon_{i} \lambda_{R, i}^{k / 2} \Gamma\left(1+\frac{k}{2}\right)
$$

in (A6) and (A7) and by varying the proportions $\left(\varepsilon_{i}\right)$ over the interval $[0,1]$ and the powers $\lambda_{R, i}$ over $[0, \infty]$. A two component Rayleigh mixture distribution taken from Abraham $^{27}$ is shown in Fig. 4.
${ }^{1}$ P. A. Crowther, "Fluctuation statistics of sea-bed acoustic backscatter," in Bottom-Interacting Ocean Acoustics, edited by W. A. Kuperman and F. B. Jensen (Plenum, New York, 1980), pp. 609-622.

${ }^{2}$ S. Stanic and E. Kennedy, "Fluctuations of high-frequency shallow-water seafloor reverberation," J. Acoust. Soc. Am. 91, 1967-1973 (1992).

${ }^{3}$ S. T. McDaniel, "Seafloor reverberation fluctuations," J. Acoust. Soc. Am. 88, 1530-1535 (1990).

${ }^{4}$ T. D. Plemons, J. A. Shooter, and D. Middleton, "Underwater acoustic scattering from lake surfaces. I. theory, experiment, and validation of the data," J. Acoust. Soc. Am. 52, 1487-1502 (1972).

${ }^{5}$ T. D. Plemons, J. A. Shooter, and D. Middleton, "Underwater acoustic scattering from lake surfaces. II. covariance functions and related statistics,” J. Acoust. Soc. Am. 52, 1503-1515 (1972).

${ }^{6}$ N. P. Chotiros, H. Boehme, T. G. Goldsberry, S. P. Pitt, R. A. Lamb, A. L. Garcia, and A. Altenburg, "Acoustic backscattering at low grazing angles from the ocean bottom: Part II. Statistical characteristics of bottom backscatter at a shallow water site," J. Acoust. Soc. Am. 77, 975-982 (1985).

${ }^{7}$ A. P. Lyons and D. A. Abraham, "Statistical characterization of highfrequency shallow-water seafloor backscatter," J. Acoust. Soc. Am. 106, 1307-1315 (1999).

${ }^{8}$ J. Dunlop, "Statistical modeling of sidescan sonar images," Proceedings of the MTS/IEEE Oceans '97, 1997, Vol. 1, pp. 33-38.

${ }^{9} \mathrm{C}$. de Moustier, "Beyond bathymetry: mapping acoustic backscattering from the deep seafloor with Sea Beam," J. Acoust. Soc. Am. 79, 316-331 (1986).

${ }^{10}$ T. K. Stanton and C. S. Clay, "Sonar echo statistics as a remote sensing tool: volume and seafloor," IEEE J. Ocean. Eng. OE-11, 79-96 (1986).

${ }^{11}$ T. K. Stanton, "Sea surface scattering: Echo peak PDF," J. Acoust. Soc. Am. 77, 1367-1369 (1985).

${ }^{12}$ T. C. Gallaudet, "Using environmental information to estimate and correct for errors in bathymetry and seafloor acoustic imagery," Chapter 3 of "Shallow water acoustic backscatter and reverberation measurements using a 68-kHz cylindrical array," Ph.D. thesis, University of California, San Diego, 2001.

${ }^{13}$ T. C. Gallaudet and C. P. de Moustier, Sea surface and volume acoustic backscatter imagery of the microbubble field of a ship's wake J. Acoust. Soc. Am. (to appear).

${ }^{14}$ T. C. Gallaudet and C. P. de Moustier, "Multibeam volume acoustic backscatter imagery and reverberation measurements in the Northeastern Gulf of Mexico," J. Acoust. Soc. Am. 112, 489-502 (2002).

${ }^{15}$ P. Faure, "Theoretical model of reverberation noise," J. Acoust. Soc. Am. 36, 259-266 (1964).

${ }^{16}$ V. V. Ol'shevskii, Characteristics of Sea Reverberation (Consultants Bureau, New York, 1967).

${ }^{17}$ D. Middleton, "A statistical theory of reverberation and similar first-order scattered fields: Part I: Waveforms and the general process," IEEE Trans. Inf. Theory IT-13, 372-392 (1967).

${ }^{18} \mathrm{D}$. Middleton, "A statistical theory of reverberation and similar first-order scattered fields: Part II: Moments, spectra, and special distributions," IEEE Trans. Inf. Theory IT-13, 393-414 (1967).

${ }^{19}$ D. Middleton, "A statistical theory of reverberation and similar first-order scattered fields: Part III: Waveforms and fields," IEEE Trans. Inf. Theory IT-18, 35-67 (1967).

${ }^{20} \mathrm{D}$. Middleton, "A statistical theory of reverberation and similar first-order scattered fields: Part IV: Statistical models," IEEE Trans. Inf. Theory IT18, 68-90 (1967)

${ }^{21}$ S. Stanic and E. G. Kennedy, "Reverberation fluctuations from a smooth seafloor," IEEE J. Ocean. Eng. OE-18, 95-99 (1993).

${ }^{22} \mathrm{H}$. Griffiths, J. Dunlop, and R. Voles, "Textural analysis of sidescan sonar imagery using statistical scattering models," in High Frequency Acoustics in Shallow Water, edited by N. G. Pace, E. Pouliquen, O. Bergem, and A. P. Lyons (NATO SACLANT Undersea Research Center, La Spezia, 1997), pp. 187-194.

${ }^{23} \mathrm{M}$. Gensane, "A statistical study of acoustic signals backscattered from the sea bottom," IEEE J. Ocean. Eng. 14, 84-93 (1989).

${ }^{24}$ W. K. Stewart, D. Chu, S. Malik, S. Lerner, and H. Singh, "Quantitative seafloor characterization using bathymetric sidescan sonar," IEEE J. Ocean. Eng. 19, 599-610 (1994).

${ }^{25}$ S. T. McDaniel, "Sea surface reverberation fluctuations," J. Acoust. Soc. Am. 94, 1551-1559 (1993).

${ }^{26}$ P. H. Dahl and W. J. Plant, "The variability of high-frequency acoustic backscatter from the region near the sea surface," J. Acoust. Soc. Am. 101, 2596-2602 (1997)

${ }^{27}$ D. A. Abraham, Modeling non-Rayleigh reverberation, SACLANT Under- 
sea Research Center Report SR-266 (SACLANT Undersea Research Center, La Spezia, June 1997). (Note: Although limited in distribution, this is available from the author upon request via email: dabraham@ieee.org).

${ }^{28}$ M. E. Frazer, "Some statistical properties of lake surface reverberation," J. Acoust. Soc. Am. 64, 858-868 (1978).

${ }^{29}$ V. C. Anderson, "Frequency dependence of reverberation in the ocean," J. Acoust. Soc. Am. 41, 1467-1474 (1967).

${ }^{30}$ T. K. Stanton, "Sonar estimates of seafloor microroughness," J. Acoust. Soc. Am. 75, 809-817 (1984).

${ }^{31}$ K. D. Ward, "Compound representation of high resolution sea clutter," Electron. Lett. 17, 561-563 (1981).

${ }^{32} \mathrm{~S}$. Watts, "Radar detection prediction in sea clutter using the compound $K$ distribution model," IEE Proc. F, Commun. Radar Signal Process. 7, 613620 (1985)

${ }^{33}$ E. Jakeman and P. N. Pusey, "A model for non-Rayleigh sea echo," IEEE Trans. Antennas Propag. 24, 806-814 (1976).

${ }^{34}$ A. Abdi and M. Kaveh, " $K$ distribution: an approximate substitue for Rayleigh-lognormal distribution in fading shadowing wireless channels," Electron. Lett. 34, 851-852 (1998).

${ }^{35} \mathrm{G}$. V. Frisk, "Intensity statistics for long-range acoustic propagation in the ocean," J. Acoust. Soc. Am. N64, 257-259 (1978).

${ }^{36} \mathrm{~B}$. Castile, "Characterization of acoustic reverberation in the ocean for high frequency, high resolution, sonar systems," Ph.D. thesis, University of California, San Diego, 1978.

${ }^{37}$ G. V. Trunk and S. F. George, "Detection of targets in non-Gaussian clutter," IEEE Trans. Aerosp. Electron. Syst. AES-6, 620-628 (1970).

${ }^{38}$ N. C. Beaulieu, A. A. Abu-Dayya, and P. J. McLane, "Estimating the distribution of a sum of independent lognormal random variables," IEEE Trans. Commun. 43, 2869-2873 (1995).

${ }^{39}$ K. Shimizu and E. L. Crow, "History, genesis, and properties," in The Lognormal Distribution: Theory and Applications, edited by E. L. Crow and K. Shimizu (Marcel Dekker, New York, 1988), Chap. 1, pp. 1-26.

${ }^{40}$ D. M. Titterington, A. F. M. Smith, and U. E. Makov, Statistical Analysis of Finite Mixture Distributions (Wiley, New York, 1985).

${ }^{41}$ T. C. Gallaudet and C. P. de Moustier, "On optimal amplitude shading for arrays of irregularly spaced or noncoplanar elements," IEEE J. Ocean. Eng. 25, 553-567 (2000).

${ }^{42}$ T. C. Gallaudet and C. P. de Moustier, "Corrections to an optimal shading for arrays of irregularly-spaced or noncoplanar ends" IEEE J. Ocean. Eng. 26-3, 416-419 (2001).

${ }^{43}$ J. S. Bendat and A. G. Piersol, Random Data: Analysis and Measurement Procedures, 2nd ed. (Wiley, New York, 1986).

${ }^{44}$ J. A. Rice, Mathematical Statistics and Data Analysis (Duxbury Press, Belmont, CA, 1995), Chap. 5, pp. 163-176.

${ }^{45}$ N. L. Johnson, S. Kotz, and N. Balakrishnan, Continuous Univariate Distributions, 2nd ed. (Wiley, New York, 1994), Vol. 1.

${ }^{46}$ M. Fisz, Probability Theory and Mathematical Statistics, 3rd ed. (Wiley, New York, 1963).

${ }^{47}$ I. T. Joliffe, "Sample sizes and the central limit theorem: The Poisson distribution as an illustration," Am. Stat. 49, 269 (1995).

${ }^{48}$ J. P. Dugan, B. W. Stalcup, and R. L. DiMarco, "Small scale activity in the upper ocean," J. Geophys. Res. Oceans 97, 5665-5675 (1992).

${ }^{49}$ J. Campbell, "The lognormal distribution as a model for bioptical variability in the sea.” J. Geophys. Res. Oceans 100, 13237-13254 (1995).

${ }^{50} \mathrm{~J}$. Aitchison and J. A. C. Brown, The Lognormal Distribution (Cambridge University Press, London, 1969).

${ }^{51} \mathrm{P}$. Greenblatt, "Distributions of volume scattering observed with an 87.5 kHz sonar,” J. Acoust. Soc. Am. 71, 879-885 (1982).

${ }^{52}$ J. R. Clark and S. Karp, “Approximations for lognormally fading optical signals,” Proc. IEEE 58, 1964-1965 (1970).

${ }^{53}$ D. Middleton, "New physical-statistical methods and models for clutter and reverberation: The KA-distribution and related probability structures," IEEE J. Ocean. Eng. 24, 261-284 (1999).

${ }^{54}$ The expressions for $\beta_{1}$ and $\beta_{2}$ in Ref. 27 contain typographical errors. Equations (A6) and (A7) are the correct forms. 